\title{
Identification of ultra-rare disruptive variants in voltage-gated calcium channel-encoding genes in Japanese samples of schizophrenia and autism spectrum disorder
}

\author{
Chenyao Wang ${ }^{1,10}$, Shin-ichiro Horigane ${ }^{2,3,10}$, Minoru Wakamori ${ }^{4,10}$, Shuhei Ueda (D) $^{2,3}$, Takeshi Kawabata ${ }^{5,6}$, Hajime Fujii ${ }^{7}$, \\ Itaru Kushima (iD ${ }^{1}$, Hiroki Kimura ${ }^{1}$, Kanako Ishizuka (iD ${ }^{1}$, Yukako Nakamura ${ }^{1}$, Yoshimi Iwayama ${ }^{8}$, Masashi Ikeda (iD ${ }^{9}$, Nakao Iwata (iD ${ }^{9}$, \\ Takashi Okada (iD) ${ }^{1}$, Branko Aleksic (D) ${ }^{\square}$, Daisuke Mori (iD) ${ }^{1}$, Takashi Yoshida ${ }^{4}$, Haruhiko Bito (iD ${ }^{7}$, Takeo Yoshikawa (iD) ${ }^{8}$, \\ Sayaka Takemoto-Kimura (iD) ${ }^{2,3 凶}$ and Norio Ozaki (iD) ${ }^{1}$
}

(c) The Author(s) 2022

Several large-scale whole-exome sequencing studies in patients with schizophrenia (SCZ) and autism spectrum disorder (ASD) have identified rare variants with modest or strong effect size as genetic risk factors. Dysregulation of cellular calcium homeostasis might be involved in SCZ/ASD pathogenesis, and genes encoding L-type voltage-gated calcium channel (VGCC) subunits Ca 1.1 (CACNA1S), $\mathrm{Ca}_{\mathrm{v}} 1.2$ (CACNA1C), Ca $\mathrm{a}_{\mathrm{v}} 1.3$ (CACNA1D), and T-type VGCC subunit $\mathrm{Ca}_{\mathrm{v}} 3.3$ (CACNA1I) recently were identified as risk loci for psychiatric disorders. We performed a screening study, using the lon Torrent Personal Genome Machine (PGM), of exon regions of these four candidate genes (CACNA1C, CACNA1D, CACNA1S, CACNA1I) in 370 Japanese patients with SCZ and 192 with ASD. Variant filtering was applied to identify biologically relevant mutations that were not registered in the dbSNP database or that have a minor allele frequency of less than 1\% in East-Asian samples from databases; and are potentially disruptive, including nonsense, frameshift, canonical splicing site single nucleotide variants (SNVs), and non-synonymous SNVs predicted as damaging by five different in silico analyses. Each of these filtered mutations were confirmed by Sanger sequencing. If parental samples were available, segregation analysis was employed for measuring the inheritance pattern. Using our filter, we discovered one nonsense SNV (p.C1451* in CACNA1D), one de novo SNV (p.A36V in CACNA1C), one rare short deletion (p.E1675del in CACNA1D), and 14 NSstrict SNVs (non-synonymous SNV predicted as damaging by all of five in silico analyses). Neither p.A36V in CACNA1C nor p. C1451* in CACNA1D were found in 1871 SCZ cases, 380 ASD cases, or 1916 healthy controls in the independent sample set, suggesting that these SNVs might be ultra-rare SNVs in the Japanese population. The neuronal splicing isoform of $\mathrm{Ca}_{\mathrm{v}} 1.2 \mathrm{with}$ the $\mathrm{p}$. $\mathrm{A} 36 \mathrm{~V}$ mutation, discovered in the present study, showed reduced $\mathrm{Ca}^{2+}$-dependent inhibition, resulting in excessive $\mathrm{Ca}^{2+}$ entry through the mutant channel. These results suggested that this de novo SNV in CACNA1C might predispose to SCZ by affecting Ca ${ }^{2+}$ homeostasis. Thus, our analysis successfully identified several ultra-rare and potentially disruptive gene variants, lending partial support to the hypothesis that VGCC-encoding genes may contribute to the risk of SCZ/ASD.

Translational Psychiatry (2022)12:84; https://doi.org/10.1038/s41398-022-01851-y

\section{INTRODUCTION}

Schizophrenia (SCZ) is a severe, chronic, and common psychiatric disorder that is characterized by psychotic symptoms such as hallucinations and delusions; the prevalence of SCZ is estimated to be $1 \%$ [1]. Autism spectrum disorders (ASDs) are a range of conditions characterized by repetitive patterns of behavior and interests, as well as persistent deficits in social communication and interaction [2]. SCZ/ASD both have been implicated to have a high heritability that is estimated as $60-90 \%$ from population-based and twin studies $[3,4]$. Recently, genomic studies coupled with large-scale collaborative projects have identified hundreds of common and rare mutations that contribute to SCZ/ASD [5-8].

Voltage-gated calcium channels (VGCCs) are transmembrane proteins that are activated by depolarization, triggering $\mathrm{Ca}^{2+}$ influx into neurons and other excitable cells [9]. VGCCs are composed of a pore-forming $a_{1}$ subunit and auxiliary $a_{2} \delta$ and $\beta$ subunits $\square$ The $a_{1}$ subunit is encoded by CACNA1 genes, including CACNA1C and CACNA1D; both of these genes have been associated with SCZ/ASD, as elucidated by several previous genetic and biological studies [5, 9-12]. Proper VGCC activity

\footnotetext{
${ }^{1}$ Department of Psychiatry, Nagoya University Graduate School of Medicine, Nagoya, Japan. ${ }^{2}$ Department of Neuroscience I, Research Institute of Environmental Medicine, Nagoya University, Nagoya, Japan. ${ }^{3}$ Molecular/Cellular Neuroscience, Nagoya University Graduate School of Medicine, Nagoya, Japan. ${ }^{4}$ Department of Oral Biology, Graduate School of Dentistry, Tohoku University, Sendai, Japan. ${ }^{5}$ Protein Research Foundation, Osaka, Japan. ${ }^{6}$ Graduate School of Frontier Biosciences, Osaka University, Osaka, Japan. ${ }^{7}$ Department of Neurochemistry, Graduate School of Medicine, The University of Tokyo, Tokyo, Japan. ${ }^{8}$ Laboratory for Molecular Psychiatry, RIKEN Brain Science Institute, Wako, Saitama, Japan. ${ }^{9}$ Department of Psychiatry, Fujita Health University School of Medicine, Toyoake, Japan. ${ }^{10}$ These authors contributed equally: Chenyao Wang, Shin-ichiro Horigane, Minoru Wakamori. ${ }^{凶}$ email: branko@med.nagoya-u.ac.jp; stakemoto@riem.nagoya-u.ac.jp
}

Received: 20 January 2022 Revised: 3 February 2022 Accepted: 9 February 2022

Published online: 26 February 2022 
underlies many essential physiological functions, governing neuronal activity, sensory functions, and muscle contraction [9]. Notably, CACNA1C and CANCA1D are two genes that are expressed predominantly in the nervous system; the encoded $\mathrm{Ca}^{2+}$ permeating $\mathrm{a}_{1}$ subunits $\left(\mathrm{Ca}_{\mathrm{v}} 1.2\right.$ and $\mathrm{Ca}_{\mathrm{v}} 1.3$, respectively) are components of channels that are located at postsynaptic somatodendritic sites [13]. Several mouse studies have provided important insights into the roles of these proteins in the pathophysiology of psychiatric disease. For example, decreased expression of $\mathrm{Ca}_{\mathrm{v}} 1.2$ in the forebrain has been shown to cause anxiety-like behavior in mice, suggesting that changes in expression or function of this protein may contribute to neuropsychiatric anxiety [12]. Timothy Syndrome is a rare multiorgan disease caused by a de novo missense mutation (p.G406R) in CANCA1C, and surviving patients may develop syndromic autism $[14,15]$. Knock-in mice expressing p.G406R CANCA1C show behavioral traits reminiscent of autistic symptoms in the social domain as well as in the repetitive/restricted behavior domain [16-18].

Recent genetic studies utilizing next-generation sequencing implicated common and rare genetic variations in VGCC-encoding genes in the etiology of psychiatric disorders such as SCZ/ASD [5, 19-26]. For instance, several large-scale genome-wide association studies (GWASs) identified CACNA1C as a critical candidate susceptibility gene in multiple psychiatric disorders, including SCZ, autism, bipolar disorder (BD), and major depression [11]. Wholeexome sequencing studies (WESs) identified recurrent de novo mutations in CACNA1D in patients with ASD [23]. The role of other VGCC-encoding genes, including CACNA1I and CACNA1S, in SCZ/ ASD remains unexplored, in spite of genetic evidence supporting the contribution of these loci to SCZ/ASD in WESs, GWASs, and genetic association studies [5, 27-29]. Furthermore, how these genetic variations associated with SCZ/ASD directly alter biophysical channel properties and causally affect SCZ/ASD pathophysiology remains unknown.

To address these issues, we report here a genetic and biological study that sought to discover possible disease-associated, rare, single-nucleotide variants (SNVs) with potentially damaging effects, in four VGCC-encoding genes, including CACNA1C, CACNA1D, CACNA11, and CACNA1S. We first sequenced the exonic regions of CANCA1C, CANCA1D, CANCA1I, and CACNA1S in Japanese patients with SCZ/ASD, and then performed association studies for prioritized variants to detect rare, potentially disruptive SNVs. Our analysis identified a new de novo SNV, p.A36V CANCA1C, in a SCZ patient. We investigated the functional consequence of this mutation on the voltage-gated calcium channel, via both in-silico three-dimensional (3D) structure prediction modeling and electrophysiological patchclamp recording. The neuronal splicing isoform of $\mathrm{Ca}_{v} 1.2$ with the $\mathrm{p}$. A36V mutation showed reduced $\mathrm{Ca}^{2+}$-dependent inhibition, presumably resulting in excessive $\mathrm{Ca}^{2+}$ entry through the mutant channel. Thus, our analysis successfully identified an ultra-rare and functionally significant variant in VGCC genes, supporting the hypothesized role of these loci in the risk of SCZ/ASD.

\section{METHODS \\ Subjects}

Three independent sample sets were used in this study. The first set, comprising samples from 370 patients with SCZ (mean $( \pm S D$ ) age $=49.73 \pm 14.75$ years; males $=52.97 \%$ ) and 192 patients with ASD (mean age $=16.34 \pm 8.36$ years; males $=77.60 \%$ ), was sequenced for rare point mutations. The second, larger set, comprising samples from 1871 patients with SCZ (mean age $=48.59 \pm 14.12$ years; males $=51.84 \%$ ), 380 patients with ASD (mean age $=18.97 \pm 9.89$ years; males $=78.48 \%$ ), and 1916 controls (mean age $=44.95 \pm 15.16$ years; males $=52.07 \%$ ), was used for association analysis of selected variants detected in the first set. For further analysis of variants detected in the first and second set, we included an independent sample set composed of 2398 patients with SCZ (mean age $=52.06 \pm 13.63$ years; males $=58.55 \%$ ) and 3679 controls (mean age $=40.60 \pm 13.42$ years; males $=39.14 \%$ ).

All participants in this study were recruited in the Nagoya University Hospital and its associated Institutes. Patients were included in the study if they met DSM-5 criteria for SCZ or ASD and were physically healthy. Healthy controls were selected from the general population and had no personal or family history of psychiatric disorders (first-degree relatives only, based on an interview with the subject). Selection was based on the following: questionnaire responses from the subjects themselves during the sample inclusion step, or an unstructured diagnostic interview conducted by an experienced psychiatrist during the blood collection step. All subjects were unrelated, lived in the central area of the Honshu island of Japan, and self-identified as members of the Japanese population. The Ethics Committees of the Nagoya University Graduate School of Medicine and Research Institute of Environmental Medicine approved this study. All experiments were performed in accordance with the Committee's guidelines and regulations. Written informed consent was obtained from all participants. In addition, each patient's capacity to provide consent was confirmed by a family member when needed. Individuals with a legal measure of reduced capacity were excluded.

\section{Resequencing and data analysis}

Genomic DNA was extracted from whole blood or saliva using the QIAGEN QIAamp DNA blood kit or tissue kit (QIAGEN, Hilden, Germany). Custom amplification primers were designed, using lon AmpliSeq Designer (Thermo Fisher Scientific, Waltham, MA, USA), to cover coding exons and flanking intron regions of the selected genes. Sample amplification and equalization were achieved using lon AmpliSeq Library Kits 2.0 and the lon Library Equalizer Kit, respectively (Thermo Fisher Scientific). Amplified sequences were ligated with Ion Xpress Barcode Adapters (Thermo Fisher Scientific). Emulsion PCR and subsequent enrichment were performed using the lon OneTouch Template Kit v2.0 on Ion OneTouch 2 and lon OneTouch ES, respectively (Thermo Fisher Scientific). The final product then was sequenced on the lon PGM sequencing platform (Thermo Fisher Scientific). Raw data output from the sequencer was deposited in the DNA Data Bank of Japan (DDBJ) (http://www.ddbj.nig.ac.jp) under Accession Number DRA004490, and uploaded to the Torrent Server (Thermo Fisher Scientific) for variant calling, with NCBI GRCh37 as a reference. The resulting VCF (variant call format) files were analyzed by Ingenuity Variant Analysis (QIAGEN) for annotation and visualization. Combined Annotation Dependent Depletion (http://cadd.gs.washington.edu/) was applied for annotation of genetic variants.

\section{Prioritization and association analysis}

Missense mutations, small insertions/deletions, and splicing site variations with a minor allele frequency $<1 \%$ were selected from the annotated data. The mutation calls then were validated for confidence by Sanger sequencing using the BigDye Terminator v3.1 Cycle Sequencing Kit (Thermo Fisher Scientific). Genotyping prioritization was based on whether the mutation was 1) located in a functional domain or motif of the protein, according to the Human Protein Reference Database (http://www.hprd. org), Pfam (http://pfam.xfam.org/), and existing literature, or functionally important, such as causing a frame shift, stop gain, or cysteine gain/loss; 2 ) a rare variant with a minor allele frequency $<1 \%$, or novel or registered in the NCBI dbSNP database (Build 137) (http://www.ncbi.nlm.nih.gov/SNP/), the 1000 Genomes Project (http://www.1000genomes.org/), the Exome Variant Server of NHLBI GO Exome Sequencing Project (ESP6500SI-V2) (http://evs.gs.washington.edu/EVS/), the Human Genetic Variation Database of Japanese genetic variation consortium (http://www.genome.med. kyoto-u.ac.jp/SnpDB), or the Integrative Japanese Genome Variation database (https://ijgvd.megabank.tohoku.ac.jp/); and 3) predicted to represent a nonsynonymous deleterious mutation by at least one of five in silico algorithms (PolyPhen2 HumDiv and HumVar [30], LRT [31], MutationTaster [32], and SIFT [33]). Additional in silico analysis, including conservation status, was performed for the prioritized SNVs using HomoloGene (http://www.ncbi.nlm.nih.gov/homologene).

Custom TaqMan SNP (single nucleotide polymorphism) genotyping assays were designed and ordered from Applied Biosystems. Allelic discrimination analysis was performed on an ABI PRISM 7900HT Sequence Detection System (Thermo Fisher Scientific). Allele and genotype frequencies of the mutations were compared between patients with SCZ and controls, or between patients with ASD and controls, using Fisher's exact test (two-tailed), with a threshold of significance set at $p<0.05$. 


\section{Identification of de novo SNVs}

We examined the inheritance pattern of the prioritized SNVs if parental samples were available. For cases with de novo SNVs, we performed paternity testing using an Identifiler plus kit for 15 short tandem repeats (AmpFISTR Identifiler plus kit, Thermo Fisher Scientific), and each parental genotype was consistent with each of their child's genotypes. The amplicons were loaded using an ABI 310 Genetic Analyzer (Thermo Fisher Scientific) and genotyped automatically using GeneMapper ID v3.2 software (Thermo Fisher Scientific). The probabilities for calculated paternity supported the conclusion that parental samples were collected from the proband's biological parents. Among parents of cases with de novo SNVs, all were psychiatrically healthy based on self-reporting or questionnaire responses.

\section{Plasmid construction and mutagenesis}

Human brain and human cerebral cortex total RNA (Takara Bio, Kusatsu, Japan) or human heart total RNA (Takara Bio) was reverse-transcribed using SuperScript IV Reverse Transcriptase (Thermo Fisher Scientific). Human CACNA1C isoforms were amplified by PCR using Platinum SuperFi DNA polymerase (Thermo Fisher Scientific) and the following primer pairs: the short and neuronal CACNA1C isoform was amplified using forward, CTCGAGCTCAAGCTTGCCACCATGCTTCGAGCCTTTGTTCAG, and reverse, CGGGCCGGTACCCTACAGGCTGCTGACGTAGA; the long and cardiac CACNA1C isoform was amplified using forward, CTCGAGCTCAAGCTTGCCACCATGCTTCGAGCCTTTGTTCAG, and reverse, CGGGCCGGTACCCTACAGGCTGCTGACGTAGACCCT. The resulting PCR fragment was inserted into a pCAG vector [34]. The clones prepared from human brain and cerebral cortex total RNA were identified as CACNA1C isoform 14 in the RefSeq database (NM_001129840.1) and used as neuronal (exon 1-containing short) isoform in this study. The clones from human heart total RNA were identified as CACNA1C isoform X30 (XM 006719017.2) and used as cardiac (exon 1a-containing long) isoform in this study. We introduced the point mutation corresponding to p.A36V into the wild-type (WT) CACNA1C by the megaprimer method [35]. The first PCR was performed with the aforementioned forward primer used for CACNA1C cloning, and a reverse primer (GGGCCAGCCCCACTGCCGCATTG) containing the mutation corresponding to $\mathrm{p.A36V}$. The second PCR was performed with the first PCR product (megaprimer) and the reverse primer used for CACNA1C cloning, and the resulting PCR product was inserted into the PCAG vector. Following construction, the identities of plasmid inserts were confirmed by sequencing.

\section{Immunoblotting}

For immunoblotting experiments, HEK293T cells were maintained in Dulbecco's Modified Eagle's Medium (DMEM; Nacalai Tesque, Kyoto, Japan) supplemented with $10 \%$ (vol/vol) heat-inactivated fetal bovine serum (FBS; Merck, Darmstadt, Germany) and Penicillin-Streptomycin Mixed Solution (Nacalai Tesque) under $5 \% \mathrm{CO}_{2}$ in air at $37{ }^{\circ} \mathrm{C}$. Cells were transfected in $35-\mathrm{mm}$ tissue culture dishes (IWAKI, Kawajiri, Japan) with $1 \mu \mathrm{g}$ of the pCAG-CACNA1CWT-IRES-EGFP (WT-Ca 1 1.2) or pCAG-CACNA1C-A36V-IRES-EGFP (A36V-Ca 1.2$)$ plasmids using X-tremeGENE 9 DNA Transfection Reagent (Roche, Basel, Germany) according to the manufacturer's instructions. Immunoblotting experiments were performed at 28 hours after transfection.

Transfected HEK293T cells were collected with ice-cold phosphatebuffered saline (PBS) and sonicated in RIPA buffer $(50 \mathrm{mM}$ Tris- $\mathrm{HCl}, \mathrm{pH} 7.5$, $150 \mathrm{mM} \mathrm{NaCl}, 0.5 \%$ sodium deoxycholate, $0.1 \%$ sodium dodecyl sulfate (SDS), 1\% NP-40) supplemented with protease inhibitor cocktail (Nacalai Tesque). After centrifugation for $5 \mathrm{~min}$ at $15,000 \times g$, the supernatant was collected and combined with $6 \times$ Sample buffer (Nacalai Tesque); the mixture then was denatured at $90^{\circ} \mathrm{C}$ (for immunoblotting of $\beta$-actin) or at room temperature (for immunoblotting of $\mathrm{Ca}_{\mathrm{v}} 1.2$ ). The extracted proteins were separated on $12 \%$ ( $\beta$-actin) or $6 \%\left(\mathrm{Ca}_{v} 1.2\right)$ SDS-polyacrylamide gels and transferred onto polyvinylidene fluoride membranes (Merck). The membranes were blocked with $5 \%$ skim milk (Nacalai Tesque) in Trisbuffered saline containing $0.05 \%$ Tween-20 (TBS-T), incubated with primary antibodies diluted in $2.5 \%$ skim milk in TBS-T overnight, and washed with TBS-T. The primary antibodies were detected with horseradish peroxidase (HRP) -conjugated secondary antibodies and ECL Prime Western Blotting Detection Reagent (GE Healthcare, Piscataway, NJ, US). Images were captured using a ChemiDoc Touch Imaging System (Bio-Rad, Hercules, CA, USA). The antibodies used for immunoblotting were purchased commercially as follows: a rabbit polyclonal antibody against $\mathrm{Ca}_{\mathrm{v}} 1.2$ (ACC-003, Alomone Labs, Jerusalem, Israel), a mouse monoclonal antibody against $\beta$-actin (A5441, Merck), and secondary antibodies conjugated to HRP (ab97051 and ab97023, abcam, Cambridge, UK).

\section{Immunocytochemistry}

For immunocytochemistry, Baby Hamster Kidney (BHK) cells were cultured in 24-well plastic tissue culture plates (IWAKI) containing $12 \mathrm{~mm}$ circular cover glasses (Glaswarenfabrik Karl Hecht, Sondheim vor der Rhön, Germany) coated with poly-L-lysine (Merck); cells were cultured in DMEM (Nacalai Tesque) supplemented with $10 \%$ (vol/vol) heat-inactivated FBS and Penicillin-Streptomycin Mixed Solution under $5 \% \mathrm{CO}_{2}$ in air at $37^{\circ} \mathrm{C}$. Cells were transfected in 24-well plastic tissue culture plates (IWAKI) with $0.2 \mu \mathrm{g}$ pCAG- $\alpha_{2} \delta-P 2 A-\beta_{3}-T 2 A-m C h e r r y-K R a s C T$ [34] and $0.05 \mu \mathrm{g}$ pCAGCACNA1C-WT or PCAG-CACNA1C-A36V plasmids using X-tremeGENE 9 DNA Transfection Reagent. After 24 hours, transfected cells were fixed for 15 min with PBS containing 4\% paraformaldehyde, permeabilized for 10 min with PBS containing $0.2 \%$ Triton X-100, and incubated for 60 min with $5 \%$ normal goat serum and $1 \%$ bovine serum albumin in PBS to block nonspecific antibody binding. After pretreatment, cells were incubated overnight with rabbit polyclonal anti-Ca 1.2 antibody (1:1000, Alomone Labs) in blocking solution, and then stained for 1 hour with an AlexaFluor 488-conjugated anti-rabbit IgG goat antibody (1:1000, Thermo Fisher Scientific) and Hoechst 33342 (Thermo Fisher Scientific) in PBS. All images were captured with a LSM 710 confocal microscope (Cael Zeiss Microscopy, Jena, Germany) equipped with a $20 \times / 0.8$ numerical aperture (NA) objective. To evaluate $\mathrm{Ca}_{\mathrm{v}} 1.2$ trafficking to the plasma membrane, we defined the regions of interest (ROIs) of the plasma membrane based on membrane-tethering red fluorescent protein (RFP-KRasCT) images using an intensity threshold. Cytoplasmic ROIs were defined as inside regions of the plasma membrane ROIs excluding nucleus regions. After background subtraction, mean $\mathrm{Ca}_{\mathrm{v}} 1.2$ intensity from each $\mathrm{ROI}$ was used to calculate $\mathrm{Ca}_{\mathrm{v}} 1.2$ intensity ratio (membrane/cytoplasm). Statistical comparison between WT and A36V mutant channels was performed by two-tailed Welch's t test.

\section{Modeling of the 3D structure of the $C a_{v} 1.2$ calcium channel and its $\mathbf{N}$-terminus}

We performed homology modeling of $\mathrm{Ca}_{\mathrm{v}} 1.2$ using three-dimensional (3D) structures homologous to $\mathrm{Ca}_{\mathrm{v}} 1.2$, as obtained from the Protein Data Bank (PDB; version 2021/11/17) using PSI-BLAST [36] with the help of the HOMCOS server [37]. We focused here on three structures: the rabbit $\mathrm{Ca}_{\mathrm{v}} 1.1$ channel (PDB ID:5gjw; 78\% protein sequence identity to $\mathrm{Ca}_{v} 1.2$ ) [38], the cockroach $\mathrm{Na}_{\mathrm{v}}$ channel (PDB ID:6a95; $32 \%$ protein sequence identity to $\mathrm{Ca}_{\mathrm{v}}$ 1.2) [39], and a complex of $\mathrm{CaM}$ and the $\mathrm{N}$-terminal spatial $\mathrm{Ca}^{2+}$ transforming element (NSCaTE)region of $\mathrm{Ca}_{\mathrm{v}} 1.2$ (PDB ID: 2 lqc; $100 \%$ protein sequence identity to $\mathrm{Ca}_{\mathrm{v}} 1.2$ ) [40]. Individual homologous template $3 \mathrm{D}$ structures are shown in Fig. S3, and the overall aligned regions are shown graphically in Fig. S1. Because the most similar structure (5gjw) does not cover the mutated residue 36 , we employed a chimeric template. We observed that the conformations of these structures were inconsistent in the N-terminal region; notably, the conformation of the NSCaTE region is $\alpha$-helical in 2lqc, but assumes a $\beta$-sheet-like structure in 6 a95 (see Figs. S2 and S3). Given that the N-terminal region (amino acids 1-89) was predicted to form an intrinsically disordered region using DISOPRED 3.16 [41], we hypothesized that the $\mathrm{N}$-terminal region might be able to assume several different conformations, and therefore decided to build models of two different structures: a folded $\mathrm{N}$-terminal structure and a CaM-binding structure. The template of the folded $\mathrm{N}$-terminal structure was generated by combining the structure $5 \mathrm{gjw}$ and the $\mathrm{N}$-terminal structure of $6 \mathrm{a} 95$ (Figs. S1, S3b). The template of the CaM-binding structure also was generated by combining the structure $5 \mathrm{gjw}$ and the $\mathrm{N}$-terminal structure of 6a95, but in this case the NSCaTE region (47-68) was taken from 2lqc chain B (Figs. S1, S3C). The combination of several structures was performed using MATRAS [42] and an in-house Python script. Using the two hybrid template structures, we successfully built two distinct models of $\mathrm{Ca}_{\mathrm{v}} 1.2$ using MODELLER 10.2 [43]. To refine the 3D structure of the model, we repeated short molecular dynamics simulations of $\mathrm{N}$-terminal domain (31-111 residues) of the 3D model using AMBER 20 [44] to generate 10 final structures, and chose one representative from these ten structures (Fig. S4). Details of the modeling are described in Supplementary information. The model of the folded $\mathrm{N}$-terminal structure is shown in Fig. $2 a$, and that of the CaM-binding structure is shown in Fig. $2 b$.

\section{Electrophysiology}

For electrophysiology, BHK cells stably expressing $a_{2} \delta$ and $\beta_{2}$ subunits $\left(B H K-a_{2} \delta+\beta_{2}\right)$, described previously [45], were used. BHK- $a_{2} \delta+\beta_{2}$ cells were cultured in DMEM (Merck) supplemented with $10 \%$ (vol/vol) heatinactivated FBS (Thermo Fisher Scientific), 30 unit/mL penicillin (Meiji Seika 
Pharma, Tokyo, Japan), and $30 \mu \mathrm{g} / \mathrm{mL}$ streptomycin (Meiji Seika Pharma) under $5 \% \mathrm{CO}_{2}$ in air at $37^{\circ} \mathrm{C}$. BHK- $\mathrm{a}_{2} \delta+\beta_{2}$ cells were seeded into Primaria $^{\mathrm{TM}}$ 60-mm cell culture dishes (Corning, Glendale, AZ, USA) and grown to $80-90 \%$ confluence. The BHK- $a_{2} \delta+\beta_{2}$ cells were used between passages 2 and 10. DNA electroporation was performed with a MicroPorator MP-100 (NanoEnTek, Seoul, South Korea) with 10- $\mu$ L tips according to the manufacturer's guidelines. For each electroporation, $1 \times$ $10^{5}$ BHK- $a_{2} \delta+\beta_{2}$ cells were suspended in Buffer $R$ and mixed with $0.5 \mu \mathrm{g}$ WT- or A36V-CACNA1C plasmid and $0.08 \mu \mathrm{g}$ EGFP-N1 (Takara Bio) vector as the marker for the transfected cells. The electroporation settings were $1,150 \mathrm{~V}, 20 \mathrm{~ms}$, and 2 pulses. Following electroporation, the cells were transferred into Primaria ${ }^{\mathrm{TM}} 35-\mathrm{mm}$ cell culture dishes (Corning). Electrophysiological experiments were performed at $3-5$ days after electroporation.

Currents were recorded using whole-cell patch-clamp techniques [46] with an EPC-10 patch-clamp amplifier (HEKA Elektronik, Reutlingen, Germany) at room temperature. Patch pipettes were made from borosilicate glass capillaries (outer diameter, $1.5 \mathrm{~mm}$; Hilgenberg, Malsfeld, Germany) using a Model P-87 Flaming-Brown micropipette puller (Sutter Instrument, Novato, CA, USA). Pipette resistance ranged from 2 to 3.5 Mohm when filled with the pipette solution described below. The series resistance was electronically compensated to $60 \%$ and both the leakage and the remaining capacitance were subtracted by $-\mathrm{P} / 5$ method. Currents were sampled at $20 \mathrm{kHz}$ after low-pass filtering at $2.9 \mathrm{kHz}$. The pipette solution contained the following components (in $\mathrm{mM}$ concentrations): Csaspartate $95, \mathrm{CsCl} 40, \mathrm{MgCl}_{2} 4$, ATP-Na 2 2, phosphocreatine- $\mathrm{Na}_{2}$ 10, EGTA 5 , and HEPES 5, and was adjusted to $\mathrm{pH} 7.2$ with $\mathrm{CsOH}$. The pipette solution for $\mathrm{Ca}^{2+}$-dependent inactivation (CDI) recording contained (in $\mathrm{mM}$ concentrations): Cs-methanesulfonate 130, CsCl 5, $\mathrm{MgCl}_{2}$ 5, ATP- $\mathrm{Na}_{2} 2$, phosphocreatine- $\mathrm{Na}_{2} 5$, EGTA 0.5 , and HEPES 10 , and was adjusted to $\mathrm{pH}$ 7.2 with $\mathrm{CsOH}$. The ' $10 \mathrm{mM} \mathrm{Ba}{ }^{2+1}$ external solution contained the following components (in $\mathrm{mM}$ concentrations): tetraethylammonium (TEA) - $\mathrm{Cl} 140$, $\mathrm{BaCl}_{2} 10$, glucose 10, and HEPES 10, and was adjusted to $\mathrm{pH} 7.4$ with TEA$\mathrm{OH}$. The ' $10 \mathrm{mM} \mathrm{Ca}^{2+\prime}$ external solution contained the following components (in $\mathrm{mM}$ concentrations): TEA-Cl $140, \mathrm{CaCl}_{2} 10$, glucose 10 , and HEPES 10, and was adjusted to $\mathrm{pH} 7.4$ with TEA-OH. Osmolarity of all solutions was approximately $300 \mathrm{mOsm}$. Rapid exchange of the external solutions surrounding the cell was provided within $1 \mathrm{sec}$ by a modified ' $Y$ tube' method [47].

The current-voltage $(I-V)$ relationship was fitted with the following equation: $I\left(V_{\mathrm{m}}\right)=\mathrm{G} *\left(\mathrm{E}_{\mathrm{rev}}-V_{\mathrm{m}}\right) /\left(1+\exp \left(\left(V_{0.5}-V_{\mathrm{m}}\right) / \mathrm{k}\right)\right.$, where $I\left(V_{\mathrm{m}}\right)$ is the peak $\mathrm{Ba}^{2+}$ current at the membrane potential of $V_{m}, G$ is the maximum conductance, $E_{r e v}$ is the apparent zero current potential in the $I-V$ relationship, $V_{0.5}$ is the potential to give a half-value of conductance, and $k$ is the slope factor that determines the steepness of the curve. To examine the voltagedependency of inactivation, currents were evoked by 20-ms test pulses to $20 \mathrm{mV}$ after 10 -ms repolarization to $-80 \mathrm{mV}$ following 2 -s conditioning membrane potential displacement from -80 to $10 \mathrm{mV}$ with $10-\mathrm{mV}$ increments. Currents were recorded every $30 \mathrm{~s}$. The curves were fitted using the Boltzmann's equation, $1 /\left(1+\exp \left(\left(V_{m}-V_{0.5}\right) / k\right)\right.$, where $V_{m}$ is the conditioning membrane potential, $V_{0.5}$ is the potential to give a halfinactivation of conductance, and $\mathrm{k}$ is the slope factor that determines the steepness of the curve. Statistical comparison between WT and A36V mutant channels was performed by a two-tailed non-paired Student's $t$ test.

\section{RESULTS}

\section{Sequencing and association study}

After sequencing of the CACNA1C, CANCA1D, CANCA11, and CACNA1S coding regions and the prioritization of sequencing data, we identified several potentially interesting variants, including 1 rare nonsense SNV (p.C1451* in CACNA1D), 1 rare short deletion ( $p$. E1675del in CACNA1D), and 14 rare NSstrict-damaging SNVs (Table 1). All mutations were confirmed using Sanger sequencing, and all of these alleles were heterozygous. Our sequence data are available in the DDBJ (http://www.ddbj.nig.ac.jp) as Accession Number DRA004490DNA. Among the identified mutations, potentially highly disruptive variants (including p.A36V in CACNA1C, p.C1451* in CACNA1D, p.E1675del in CACNA1D, and NSstrict SNV p.G330R in CACNA1D) were genotyped in an independent sample set comprising 1871 SCZ cases, 380 ASD cases, and 1916 controls (Table 2). Neither p.A36V in CACNA1C nor p.C1451* in CACNA1D were found in SCZ/ASD cases or healthy controls in the independent sample set, suggesting that these SNVs might be ultra-rare SNVs in the Japanese population. p.E1675del CACNA1D was found in both SCZ/ASD cases and healthy controls, but frequencies in psychiatric patients and healthy controls did not exhibit a statistically significant difference. $p$. G330R CACNA1D was found in SCZ/ASD cases, but not in healthy controls, suggesting that this mutation might constitute a significant SNV that is enriched exclusively in patients with psychiatric disorders. Therefore, an additional association study was performed in a much larger independent sample set comprising 4255 SCZ cases and 5565 controls. This analysis revealed that p.G330R CACNA1D was found in both SCZ cases and healthy controls, but frequencies in psychiatric patients and healthy controls did not exhibit a statistically significant difference (Table 3).

\section{Identification of a de novo SNV}

For the rare and potentially function-damaging variants discovered in our sequencing study, we attempted a check of their inheritance pattern if parent or sibling samples were available. We successfully discovered one de novo SNV (p.A36V in CACNA1C) that was present only in the proband (i.e., parents or siblings did not carry the mutation). The patient with the de novo SNV (p.A36V in CACNA1C) was diagnosed with $\mathrm{SCZ}$ at the age of 23. In the outpatient clinic, the patient showed additional symptoms of SCZ, including irritability and soliloquy. The symptoms were controlled well by risperidone $(4 \mathrm{mg}$ per day). The patient was not diagnosed with any diseases other than SCZ. This individual's electrocardiogram test was normal. The patient had a sibling who also was diagnosed with $\mathrm{SCZ}$, although we lack detailed clinical information for this individual; neither parent exhibited any psychiatric disorder.

\section{The p.A36V mutation does not show apparent deficits in membrane trafficking by $\mathrm{Ca}_{\mathrm{v}} \mathbf{1 . 2}$ channels}

To assess the biological significance of the A36V mutation in $\mathrm{Ca}_{\mathrm{v}} 1.2$ channels expressed in the brain, we constructed an A36V $\mathrm{Ca}_{\mathrm{v}}$ 1.2-encoding plasmid vector. The CACNA1C gene includes alternative exons designated exon 1 (short and neuronal) and exon 1a (long and cardiac); we cloned a 'short' isoform cDNA containing the alternative exon 1 from adult human brain total RNA. Six clones were obtained in total, and all clones were identified as transcription variant 14 in the RefSeq database (NM_001129840.1). We introduced the mutation encoding the $\mathrm{A} 36 \mathrm{~V}$ substitution into the plasmid encoding this neuronal isoform, which typically would be expressed predominantly in adult human brain (Fig. 1c). An A39V mutation, located in the vicinity of $\mathrm{A} 36 \mathrm{~V}$ (Fig. $1 \mathrm{a}, \mathrm{b}$ ) in $\mathrm{Ca}_{\mathrm{v}} 1.2$ channels, previously was linked to Brugada syndrome, which presents with atrial fibrillation and abbreviated QT interval. A cardiac 'long' isoform of $\mathrm{Ca}_{\mathrm{v}} 1.2$ containing both the alternative exon $1 \mathrm{a}$ and the A39V mutation has been shown to induce a membrane trafficking defect and to exhibit drastically decreased $\mathrm{Ca}^{2+}$ currents [48]. Therefore, we examined whether membrane trafficking of neuronal $\mathrm{Ca}_{v} 1.2$ channels harboring the A36V mutation is defective. When WT and $A 36 V \mathrm{Ca}_{\mathrm{v}} 1.2$ neuronal channels were expressed with $\mathrm{a}_{2} \delta$ and $\beta_{3}$ auxiliary subunits in BHK cells, we found that the two forms accumulated to similar levels at the plasma membrane regions (Fig. 1d-f). This result is consistent with a prior observation that A39V Brugada mutation in the neuronal $\mathrm{Ca}_{\mathrm{v}} 1.2$ isoform did not exhibit apparent membrane trafficking deficits [49]. These data suggested that a general loss of membrane expression is unlikely to account for the neuronal phenotype of the de novo A36V mutation in $\mathrm{Ca}_{\mathrm{v}} 1.2$.

\section{The A36V mutation may alter conformational equilibrium between folded and CaM-binding structures at the $\mathrm{Ca}_{\mathrm{v}} \mathbf{1 . 2} \mathrm{N}$ - terminus, thereby modulating CDI}

We next tested whether the A36V mutation modulated the biophysical properties of the $\mathrm{Ca}_{\mathrm{v}} 1.2$ protein via alteration of structural dynamics of the alpha1 subunit conformations. $\mathrm{Ca}_{\mathrm{v}} 1.2$ 


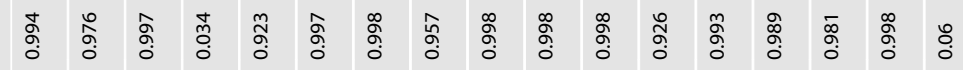

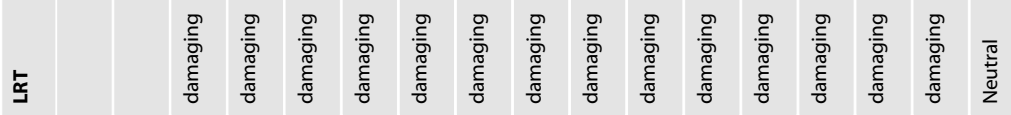

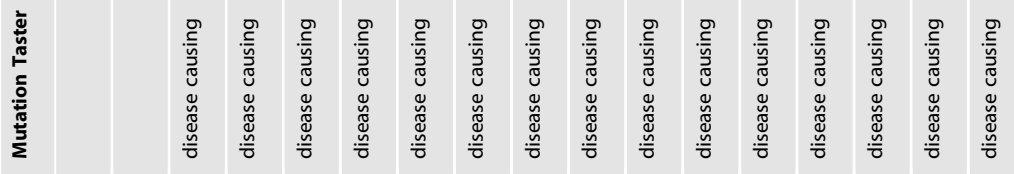

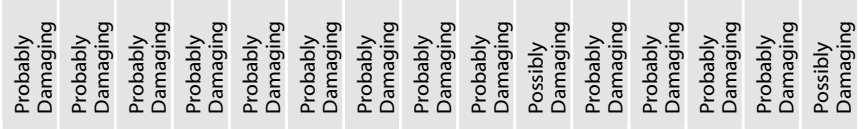

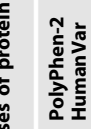

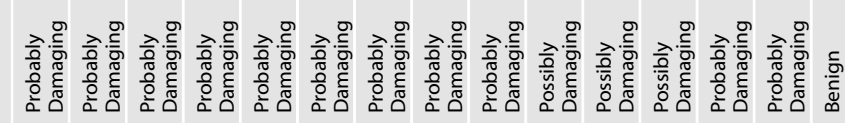

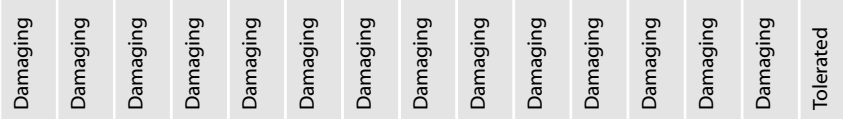

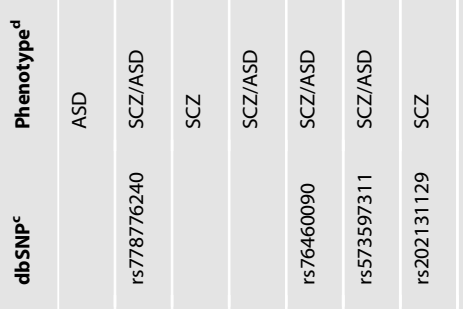

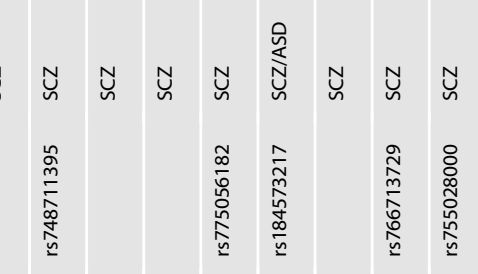

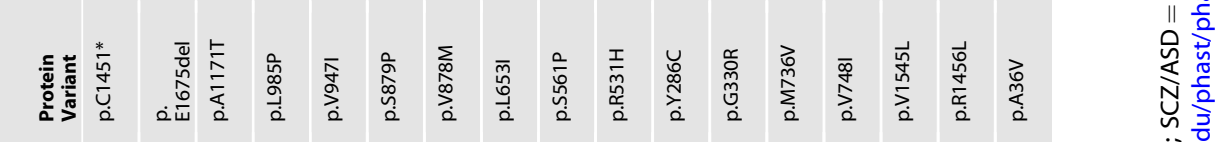

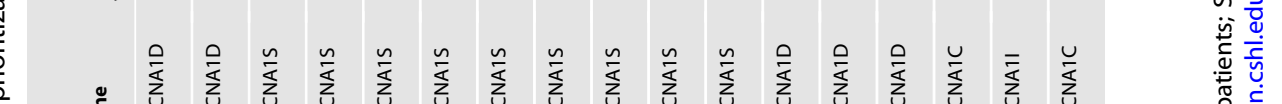

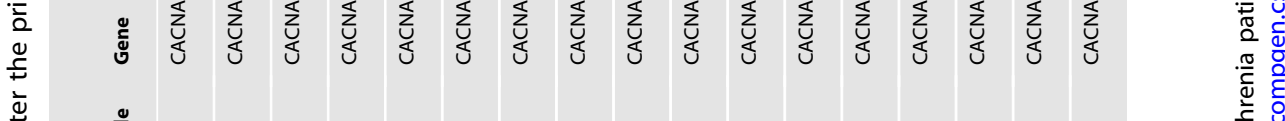

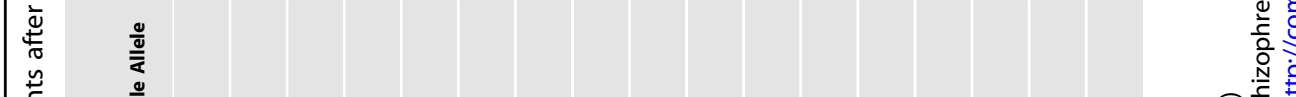

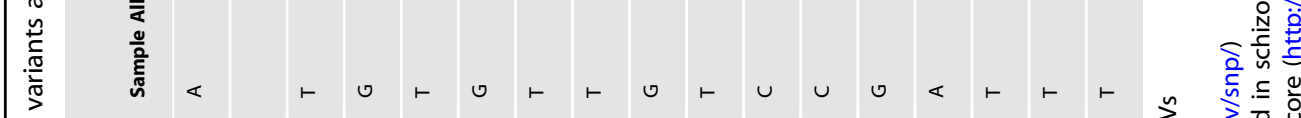

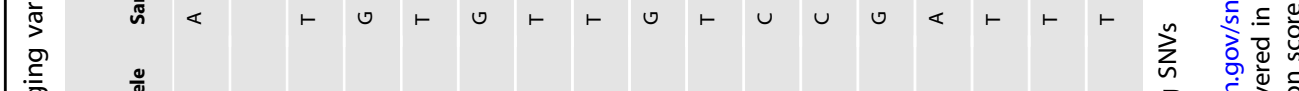

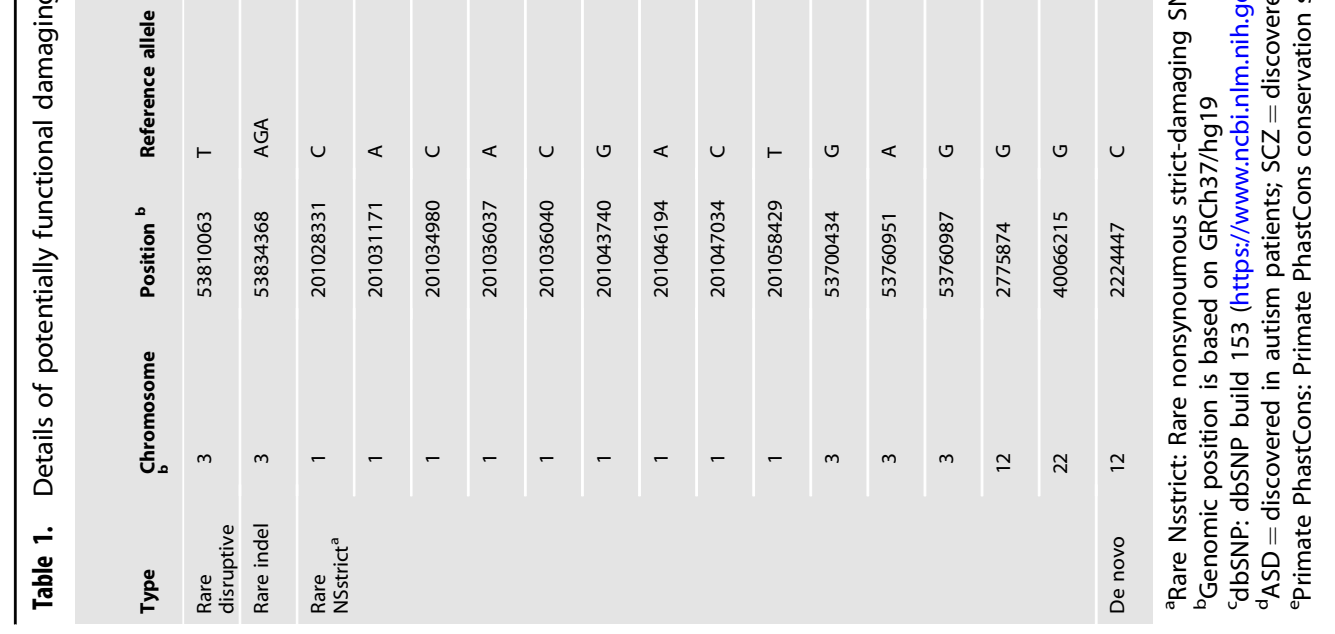


has multiple CaM-binding sites in its $\mathrm{N}$-terminus and $\mathrm{C}$-terminus. $\mathrm{Ca}^{2+}$ ions entering through $\mathrm{Ca}_{\mathrm{v}} 1.2$ pore bind to $\mathrm{CaM}$, and consequently cause $\mathrm{CDI}$ [50-53]. We noticed that the A36V mutation is located close to the NSCaTE region, a CaM interaction site that has been shown to be necessary for $\mathrm{N}$-lobe dependent CDI $[50,53]$. Using homologous 3D-structures, we modeled two different structures of $\mathrm{Ca}_{\mathrm{v}} 1.2$ (a folded $\mathrm{N}$-terminal structure and an open (aM-binding N-terminal structure), and assumed the conformational ensemble of $\mathrm{Ca}_{\mathrm{v}} 1.2$ is in an equilibrium among various structures including these two structures (Fig. 2). The $36^{\text {th }}$ residue of $C a_{v} 1.2$ (alanine in the WT) have hydrophobic contacts with other nonpolar residues (A39 and A97) in the folded $\mathrm{N}$-terminal structure (Fig. 2a, c), whereas this residue is more exposed to solvent in the CaM-binding structure (Fig. 2b). Because valine is more hydrophobic than alanine, the models suggested that the A36V substitution may shift the conformational equilibrium toward the folded $\mathrm{N}$-terminal structure, thereby decreasing the frequency of formation of the CaM-binding structure at the NSCaTE region of $\mathrm{Ca}_{\mathrm{v}} 1.2$ (Fig. $2 \mathrm{~d}$ ).

To determine the electrophysiological characteristics of the A36V Ca 1.2 channels, we recorded whole-cell $\mathrm{Ba}^{2+}$ currents through recombinant $\mathrm{Ca}_{\mathrm{v}} 1.2$ channels transiently expressed in $B H K-a_{2} \delta+\beta_{2}$ cells [45]. The current-voltage (I-V) relationship for the neuronal A36V Cav1.2 channel was similar to that for the WT channel. The peak current density (peak current amplitude divided by cell capacitance) for the neuronal $\mathrm{A} 36 \mathrm{~V} \mathrm{Ca} \mathrm{v}_{\mathrm{v}} 1.2$ channel $(15.8 \pm$ $2.0 \mathrm{pA} / \mathrm{pF}, n=18)$ did not differ significantly from that of the neuronal WT $\mathrm{Ca}_{\mathrm{v}} 1.2$ channel $(19.8 \pm 2.9 \mathrm{pA} / \mathrm{pF}, n=12)($ Fig. 3a, b), consistent with a lack of a mutation effect on channel membrane trafficking (Fig. 1d-f). The steady-state inactivation curves for both channels were very similar (Fig. $3 \mathrm{c}$ ). These results suggest that the A36V mutation does not change the voltage dependency of activation and inactivation of the neuronal $\mathrm{Ca}_{\mathrm{v}} 1.2$ channel.

Finally, we examined the effect of A36V on global CDI. This parameter was tested with the use of the pipette solution containing $0.5 \mathrm{mM}$ EGTA. We recorded $\mathrm{Ba}^{2+}$ and $\mathrm{Ca}^{2+}$ currents at $30 \mathrm{mV}$ from the same BHK cell expressing neuronal $\mathrm{Ca}_{\mathrm{v}} 1.2$ (Fig. $3 d-f) . \mathrm{Ba}^{2+}$ currents decayed slowly, but $\mathrm{Ca}^{2+}$ currents decayed completely within $350 \mathrm{msec}$ (Fig. $3 \mathrm{~d}$ ). The ratio of remaining current amplitude to the peak current amplitude was calculated at different time points after depolarization to $30 \mathrm{mV}$ (Fig. 3e, f). $\mathrm{Ca}^{2+}$ currents (Fig. 3f), but not $\mathrm{Ba}^{2+}$ currents (Fig. 3e) of neuronal A36V $\mathrm{Ca}_{\mathrm{v}} 1.2$, were reduced mildly, but significantly more slowly, than $\mathrm{Ca}^{2+}$ currents of the neuronal WT $\mathrm{Ca}_{\mathrm{v}} 1.2$, suggesting that the A36V mutation affects global $C D I$ in neurons. We further examined the impact of $\mathrm{A} 36 \mathrm{~V}$ in the cardiac isoform that contain the longer $\mathrm{N}$-terminal region encoded by exon $1 \mathrm{a}$ (Fig. $3 \mathrm{~g}-\mathrm{i}$ ). In contrast, $\mathrm{Ca}^{2+}$ currents (Fig. 3i) and $\mathrm{Ba}^{2+}$ currents (Fig. 3h) of cardiac A36V Ca 1.2 decayed similarly to those of cardiac WT $\mathrm{Ca}_{\mathrm{v}} 1.2$. The reported electrophysiological properties of the A39V Brugada mutation in a neuronal isoform is more complex [54], suggesting co-existing overlapping pathophysiology of the A39V mutation, especially in cardiac mutant channels. Taken together, these data indicated that $\mathrm{A} 36 \mathrm{~V}$ neuronal $\mathrm{Ca}_{\mathrm{v}} 1.2$ is a mild gain-of-function mutant channel, while the A36V cardiac $\mathrm{Ca}_{\mathrm{v}} 1.2$ behaves similarly to the WT channel, consistent with the relatively late onset of SCZ symptoms and normal cardiac functions.

\section{DISCUSSION}

Recent large-scale genetic studies have reported that ultra-rare disruptive SNVs are highly enriched in patients with SCZ/ASD, especially SNVs in sets of VGCC genes, providing an opportunity to discover the cellular processes that are relevant to the pathogenesis of SCZ/ASD [5, 11, 22, 23, 55]. However, the cellular processes whereby these rare disruptive SNVs give rise to psychiatric disorders remain unknown. To address this question, we performed a comprehensive search, including a genetic and 
a

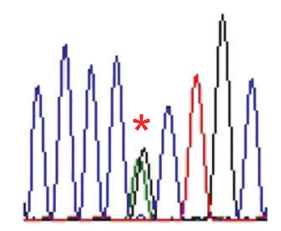

c.C107T > p.A36V

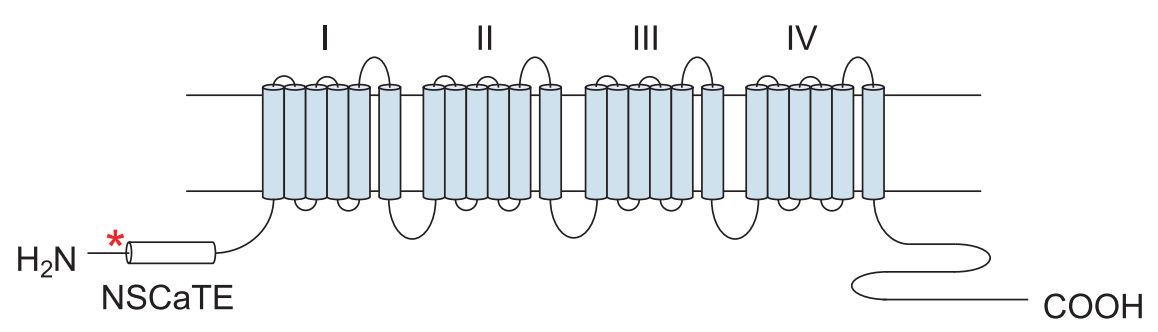

NSCaTE

$\mathrm{Ca}_{\mathrm{v}} 1.2 \mathrm{WT}$

$\mathrm{Ca}_{\mathrm{v}} 1.2 \mathrm{~A} 36 \mathrm{~V}$

MVNENTRMYIPEENHQGSNYGSPRPAHANMNANAAAGLAPEHIPTPGAALSWQAAIDAARQAKLMGSA MVNENTRMYIPEENHQGSNYGSPRPAHANMNANAAVGLAPEHIPTPGAALSWQAAIDAARQAKLMGSA

$\mathrm{Ca}_{v} 1.2 \mathrm{~A} 39 \mathrm{~V}$ (Brugada) MVNENTRMYIPEENHQGSNYGSPRPAHANMNANAAAGLVPEHIPTPGAALSWQAAIDAARQAKLMGSA
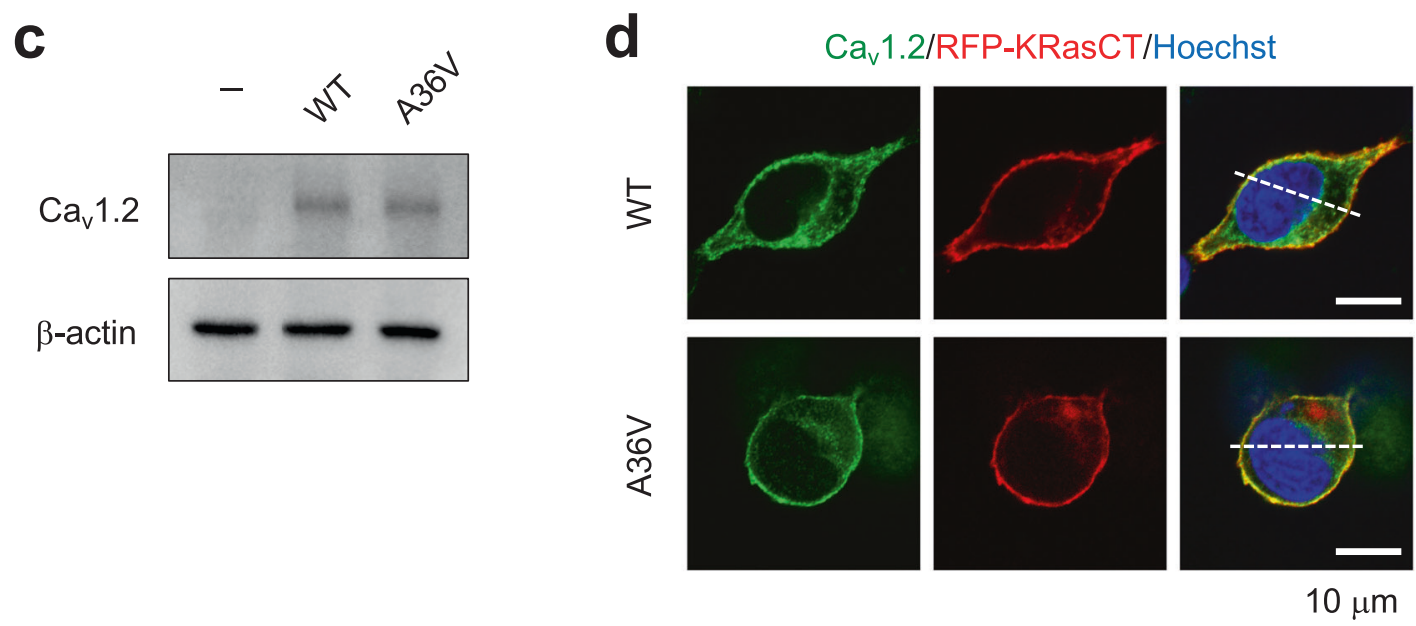

e
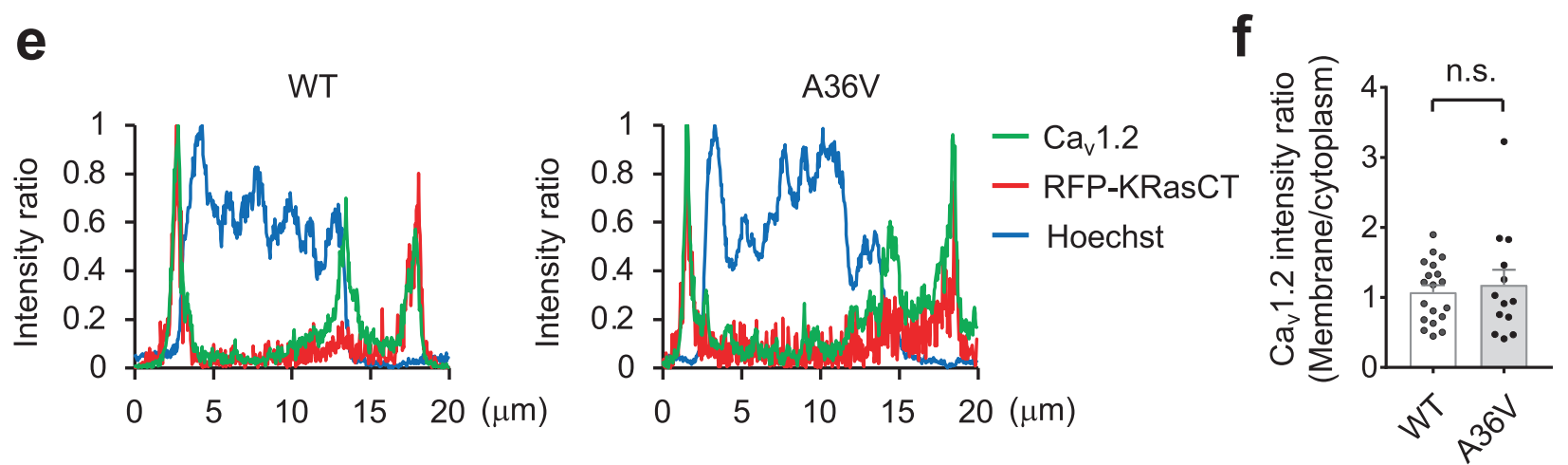

Fig. 1 The A36V mutation does not affect membrane localization of $\mathbf{C a}_{\mathbf{v}} \mathbf{1 . 2}$ channels. a Sanger sequencing results for the de novo variant $\mathrm{p}$. A36V (left) and schematic illustration of the primary structure of the $\mathrm{Ca}_{\mathrm{v}} 1.2$ channel (right). The red asterisk indicates the A36V mutation near the N-terminal spatial $\mathrm{Ca}^{2+}$-transforming element (NSCaTE). b The N-terminal amino acid sequences for Ca 1.2 channels (short isoforms). The A36V mutation and the A39V Brugada mutation are indicated in red letters. c Expression of wild-type (WT) and A36V Ca 1.2 channels in HEK293T cells as detected by anti-Ca 1.2 antibody. $\mathbf{d}$ Membrane localization of WT and A36V Ca 1.2 channels overexpressed in BHK cells. The plasma membrane was visualized by membrane-tethering red fluorescent protein (RFP-KRasCT). e The fluorescence intensity profiles of the line shown in Fig. 1d. f Plasma membrane to cytoplasm intensity ratio of $\mathrm{Ca}_{\mathrm{v}} 1$.2. Statistical comparison was performed by two-tailed Welch's $t$ test (n.s., not significant). Data are presented as mean \pm s.e.m.

functional analysis of VGCC genes. Our analysis identified 1 nonsense SNV (p.C1451* in CACNA1D), 1 de novo SNV (p.A36V in CACNA1C), 1 rare short deletion (p.E1675del in CACNA1D), and 14 NSstrict SNVs (Non-synonymous SNV predicted as damaging by all of five in silico analyses). Neither p.A36V CACNA1C nor p.C1451* CACNA1D were found in 1871 SCZ cases, 380 ASD cases, or 1916 healthy controls in the independent sample set, suggesting that these SNVs might be ultra-rare SNVs in the Japanese population. p.G330R CACNA1D was detected in both SCZ and healthy controls, at frequencies that did not show statistically significant differences between the two groups. However, it should be noted that the sample size was too small to completely rule out the possibility of association. The p.G330R mutation is located in the S5-S6 linker of repeat I of the encoded protein; this region previously was shown 


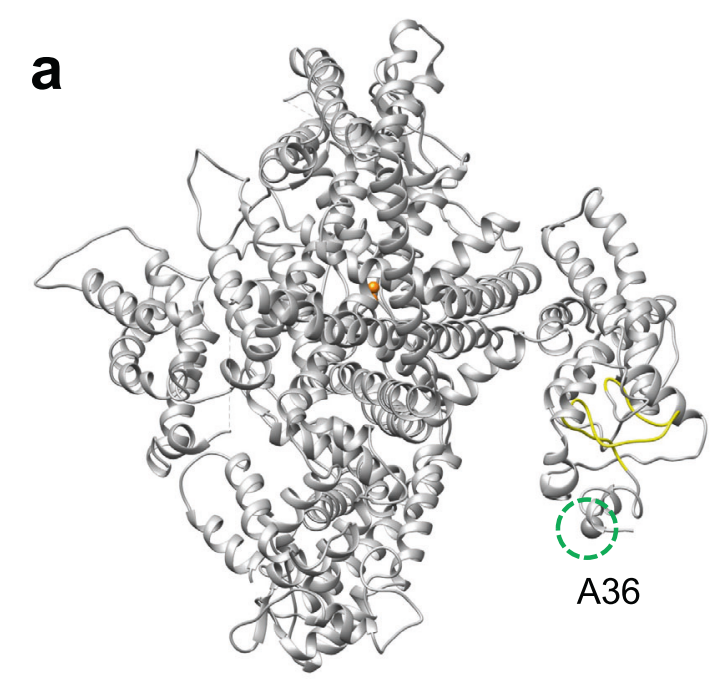

C

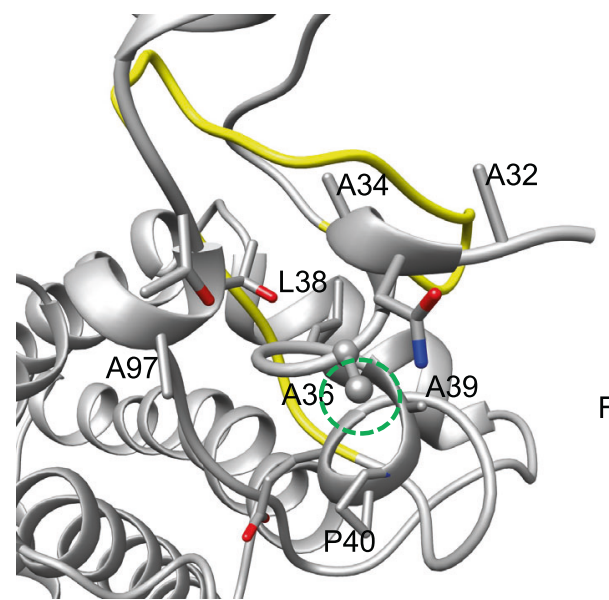

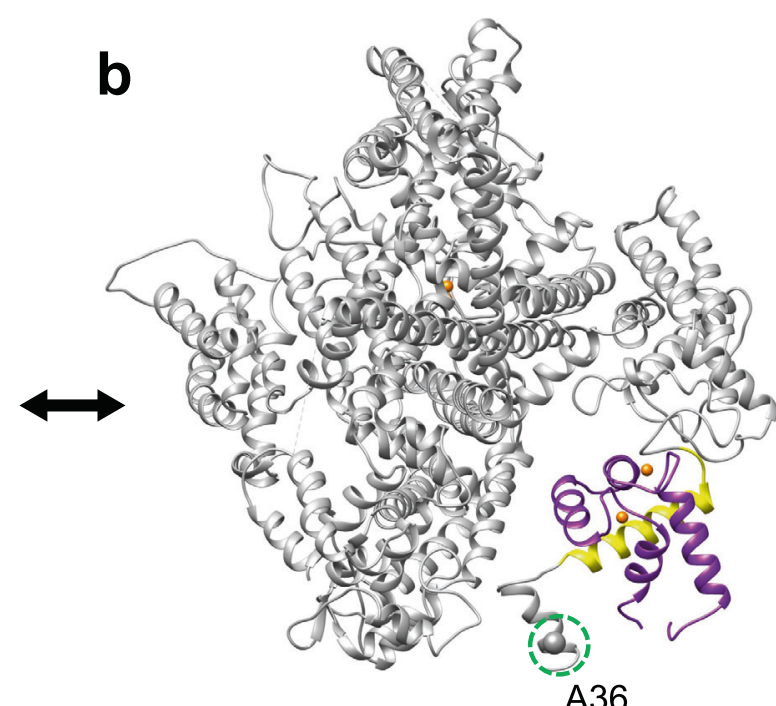

A36

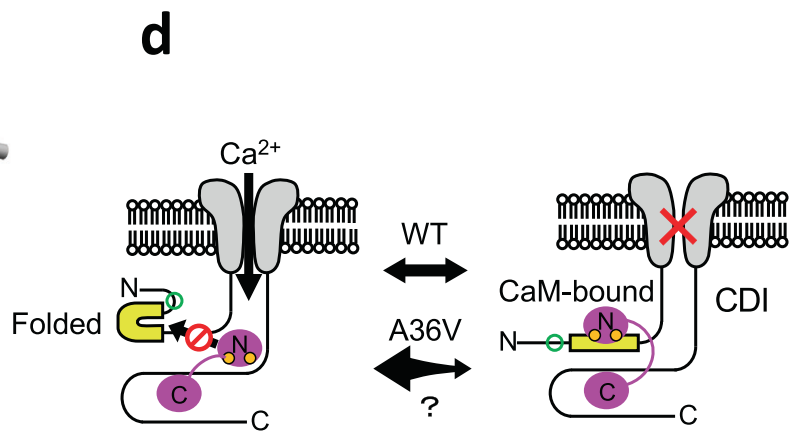

Fig. 2 Conformational equilibrium between the folded $\mathrm{N}$-terminal structure and calmodulin (CaM) -binding structure of $\mathrm{Ca}_{\mathrm{v}} \mathbf{1 . 2}$. a Threedimensional (3D) model structure of the $\mathrm{Ca}_{\mathrm{v}} 1.2$ channel with the folded N-terminal structure. $\mathbf{b}$ 3D model structure of the Cav 1.2 channel with the CaM-binding structure, shown in complex with the N-lobe of CaM. c Enlarged view around the alanine 36 (A36) residue of the folded $\mathrm{N}$-terminal structure. The $\mathrm{A} 36$ site is highlighted by green dotted circles. The $\mathrm{N}$-terminal spatial $\mathrm{Ca}^{2+}$-transforming element (NSCaTE) region (47-68), $\mathrm{Ca}^{2+}$, and CaM are indicated in yellow, orange, and magenta, respectively. Molecular graphics were created using UCSF Chimera [74]. d A schematic illustration of the hypothesis that the A36V mutation attenuates $\mathrm{Ca}^{2+}$-dependent inactivation (CDI) by conformational equilibrium shift favoring the folded structure.

to be a critical determinant of L-type VGCC conductance [56], suggesting that this mutation may have biological significance. To assess the effect of the A36V mutation on $\mathrm{Ca}_{\mathrm{v}} 1.2$ channel properties, we performed in silico $3 \mathrm{D}$ structural prediction modeling and electrophysiological studies. The modeling analysis led to a previously unexplored (to our knowledge) hypothesis, that the $\mathrm{N}$-terminal region of $\mathrm{Ca}_{\mathrm{v}} 1.2$ exists in an equilibrium between two alternative conformations, a folded $\mathrm{N}$-terminal structure and an open CaM-binding structure. We postulated that the A36V mutation of neuronal $\mathrm{Ca}_{\mathrm{v}} 1.2$ may shift the conformational equilibrium toward the folded $\mathrm{N}$-terminal structure, thereby modulating the $\mathrm{CDI}$ of neuronal $\mathrm{Ca}_{\mathrm{v}} 1.2$ channels. Consistent with this hypothesis, we observed a decrease in the CDI of $A 36 \mathrm{~V}$ neuronal $\mathrm{Ca}_{\mathrm{v}} 1.2$ channels. These results support the proposal that the newly identified $\mathrm{A} 36 \mathrm{~V}$ mutation affects channel properties in a mild gain-of-function fashion, potentially explaining changes in pathophysiology underlying SCZ.

Our analysis identified a new de novo SNV, p.A36V CACNA1C, in a SCZ patient; we therefore investigated the functional consequence of this mutation on the function of the voltage-gated calcium channel, via both in silico 3D structure prediction modeling and electrophysiological patch-clamp recording. Modeling analysis suggested a possible shift in the dynamic equilibrium of $\mathrm{A} 36 \mathrm{~V}$
$\mathrm{Ca}_{\mathrm{v}} 1.2$ 's N-terminus, disfavoring a calmodulin (CaM) -bound structure. Consistent with this modeling, A36V neuronal $\mathrm{Ca}_{v} 1.2$ showed a significant reduction in CDI compared to WT neuronal $\mathrm{Ca}_{\mathrm{v}} 1.2$, with little change in voltage-dependent activation and inactivation. $C D I$ is a negative feedback mechanism limiting excessive $\mathrm{Ca}^{2+}$ entry $[51,57]$; therefore, $\mathrm{A} 36 \mathrm{~V}$ can be regarded as a gain-of-function mutation in neuronal $\mathrm{Ca}_{\mathrm{v}} 1.2$. Thus, our analysis successfully identified an ultra-rare and functionally significant variant in VGCC-encoding genes, supporting the hypothesized role of these loci in the risk of SCZ/ASD, and indicating that a mild gainof-function in $\mathrm{Ca}_{\mathrm{v}} 1.2$ may play a role in the etiology of SCZ.

A series of GWAS and WES studies have suggested causal links between common and rare variants of CACNA1C and psychiatric disorders including SCZ, BD, and ASD [19]. Growing evidence has shown that gain-of-function mutations in $\mathrm{Ca}_{\mathrm{v}} 1.2$ are associated with psychiatric disorders. For instance, a CACNA1C lesion causing a G406R substitution in the encoded protein was identified in patients with Timothy syndrome; multiple studies have shown that this gain-offunction mutation is associated with prolonged QT intervals, syndactyly, and ASD $[14,15]$. Furthermore, the CACNA1C gene polymorphism rs1006737, known to be associated with SCZ and BD, has been shown to augment $\mathrm{Ca}_{\mathrm{v}} 1.2$ channel activity and CACNA1C mRNA expression in induced human neurons and in human brain 
a

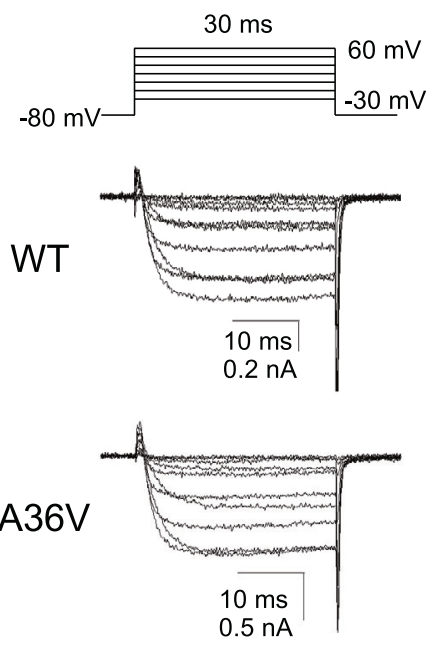

d

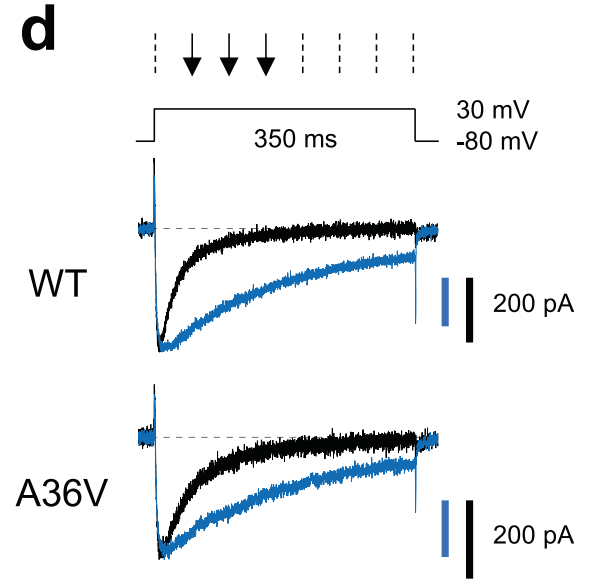

WT

h

g
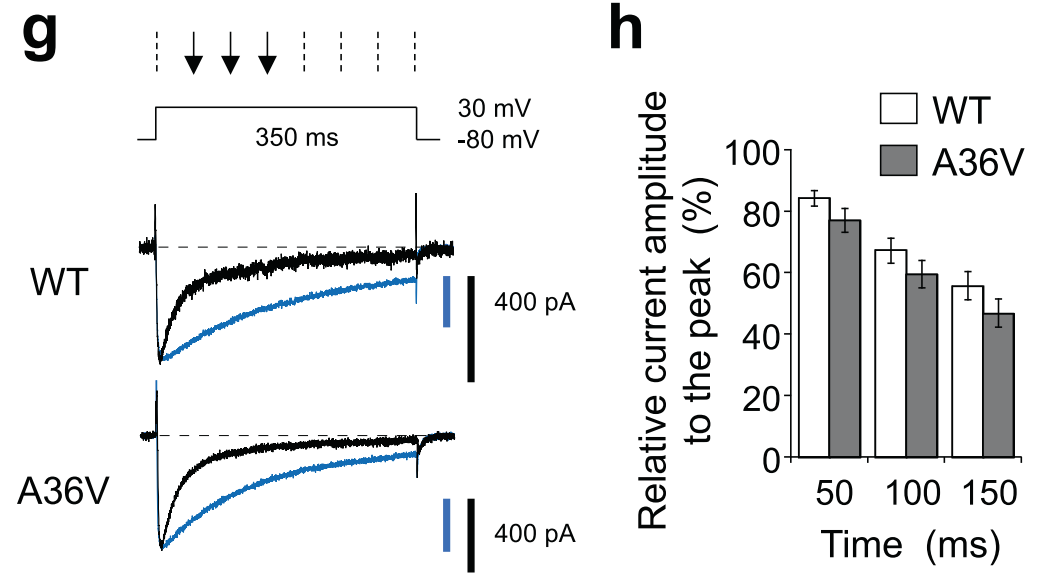

e
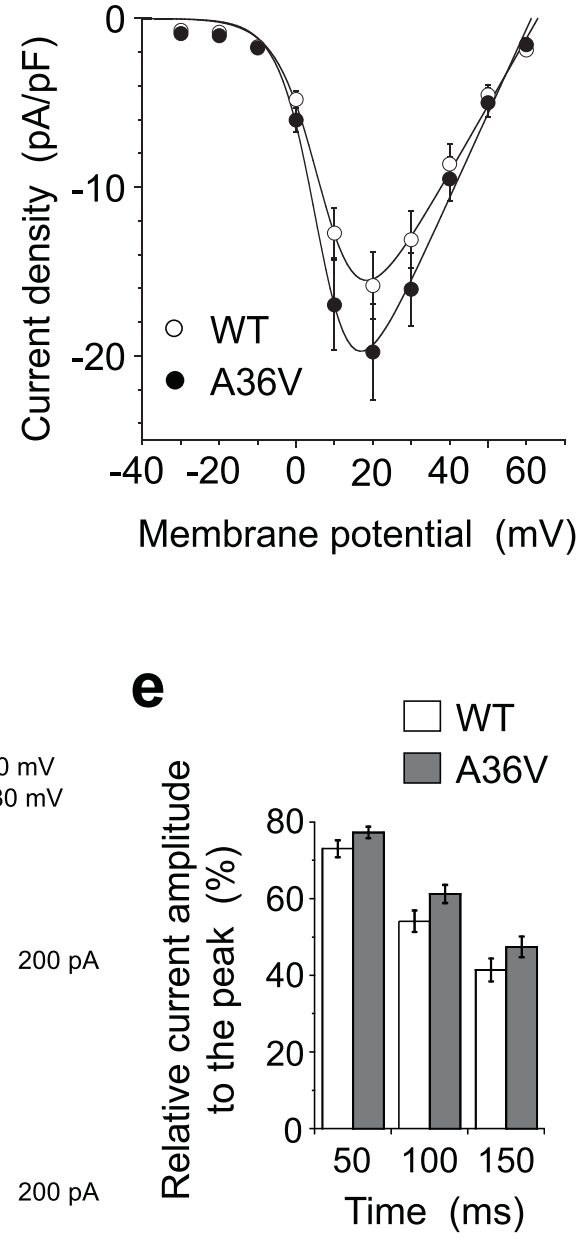

f

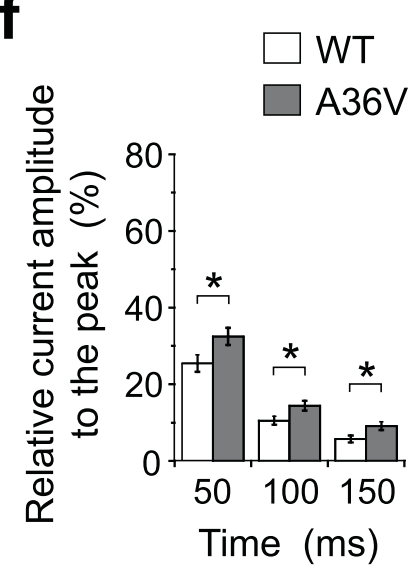

$[58,59]$. Intriguingly, the Brugada syndrome $A 39 \mathrm{~V} \mathrm{Ca} \mathrm{Ca}_{\mathrm{v}} 1.2$ mutation, when encoded in a long splicing isoform (exon 1a-containing cardiac type), was shown to function as a loss-of-function mutation with impaired membrane trafficking [48]; when encoded in a short splicing isoform (exon 1-containing neuronal type), this change possibly functions as a gain-of-function mutation by modulating CDI
C

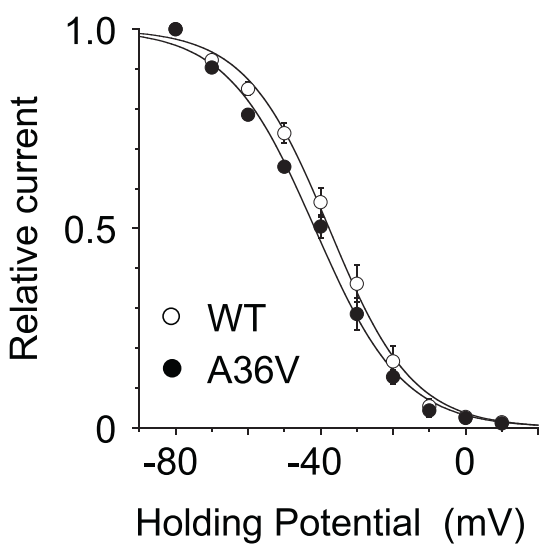

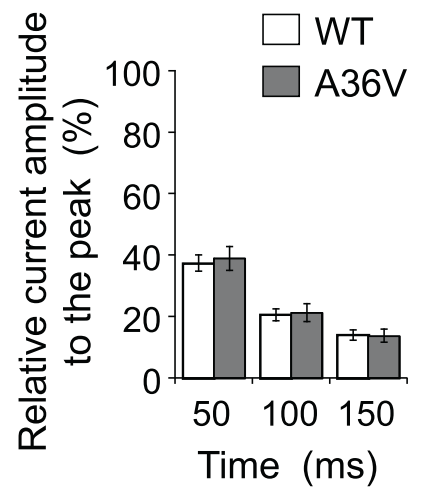

[54]. These splicing isoform-specific observations suggest that the presence of the same $\mathrm{Ca}_{\mathrm{v}} 1.2$ mutation in separate isoforms leads to distinct outcomes.

The present study showed that the A36V mutation, when carried by the short neuronal isoform, inhibits $\mathrm{CDI}$, suggesting that $\mathrm{A} 36 \mathrm{~V}$ and $\mathrm{A} 39 \mathrm{~V}$, which are located near the NSCaTE domain in 
Fig. 3 Electrophysiological properties of p.A36V Cav 1.2 channels. a Families of $\mathrm{Ba}^{2+}$ currents evoked by 30-ms depolarizing pulses from -30 to $60 \mathrm{mV}$ with increments of $10 \mathrm{mV}$ for wild-type (WT) and A36V neuronal $\mathrm{Ca}_{\mathrm{v}} 1.2$ channels. b Current density-voltage (I-V) relationships. Data are expressed as mean \pm s.e.m., WT: $n=18, \mathrm{~A} 36 \mathrm{~V}: n=12$. The values of G, Erev, $\mathrm{V}_{0.5}$, and k were $-0.40,63.0 \mathrm{mV}, 7.6 \mathrm{mV}$, and 5.6 mV for WT channels, and $-0.50,61.3 \mathrm{mV}, 6.7 \mathrm{mV}$, and $4.9 \mathrm{mV}$ for A36V Cav1.2 channels. c Inactivation curves for WT $(\mathrm{O}, n=9)$ and $\mathrm{A} 36 \mathrm{~V}(\mathbf{0}, n=4)$ neuronal $\mathrm{Ca}_{\mathrm{v}} 1.2$ channels. Data are expressed as mean \pm s.e.m. The values of $\mathrm{V}_{0.5}$, and $\mathrm{k}$ were (respectively) $-37.6 \mathrm{mV}$ and $11.5 \mathrm{mV}$ for $\mathrm{WT}$ channels, and $-41.6 \mathrm{mV}$ and $12.1 \mathrm{mV}$ for A36V Ca 1.2 channels. $\mathbf{d}, \mathbf{g ~ C a}{ }^{2+}$-dependent inactivation (CDI) of neuronal (d) and cardiac (g) Ca 1.2 channels. $\mathrm{Ba}^{2+}$ (blue) and $\mathrm{Ca}^{2+}$ (black) currents evoked by $350-\mathrm{ms}$ step depolarization to $30 \mathrm{mV}$ were normalized at their peak current amplitudes for WT and A36V Ca 1.2 channels. e, f, h, i, Ratios of current amplitude to the peak amplitude were plotted against depolarizing time in the $\mathrm{Ba}^{2+}(\mathbf{e}, \mathbf{h})$ and the $\mathrm{Ca}^{2+}(\mathbf{f}, \mathbf{i})$ external solutions. The numbers of recorded cells were 10 and 15 for WT and A36V neuronal Ca 1.2 channels $(\mathbf{e}, \mathbf{f})$, and 8 and 6 for WT and A36V cardiac Ca 1.2 channels (h-i), respectively. Statistical comparison was performed by two-tailed non-paired Student's $t$ test $\left({ }^{*} p<0.05\right)$. Data are presented as mean \pm s.e.m.

$\mathrm{Ca}_{\mathrm{v}} 1.2$, both serve as mild gain-of-function mutations in the neuronal isoform. In silico 3D structural modeling indicated that these residues form part of a hydrophobic core in the $\mathrm{N}$-terminal folded structure, but are exposed in the CaM-binding structure (Fig. 2). These substitutions with more hydrophobic residues may stabilize the $\mathrm{N}$-terminal folded structure while destabilizing the CaM-binding structure, which in turn could affect the CDI. Given that the A36V mutation was identified from an individual with SCZ without cardiac symptoms, this mutation may not influence the activity of cardiac channels. Consistent with this notion, it was found that A36V mutation did not affect electrophysiological properties when we tested the mutation in the cardiac isoform. $\mathrm{Ca}_{\mathrm{v}} 1.2$ has multiple isoforms with different functional properties and tissue-selective expression [60, 61]. To understand the complexity of the contribution of $\mathrm{A} 36 \mathrm{~V}$ and other mutations to psychiatric disorders and cardiac arrhythmia, future studies will need to consider cell type (neuronal or cardiac), $\mathrm{Ca}_{\mathrm{v}} 1.2$ splicing isoform, and co-expressed auxiliary subunits that contribute to the functional diversity of $\mathrm{Ca}_{\mathrm{v}} 1.2$ channels [62].

Trios-based exome sequencing studies have supported the hypothesis that de novo SNVs, especially de novo SNVs in proteincoding sequences, are related to increased risk for psychiatric disorders such as SCZ/ASD and BD [63-66]. Furthermore, a relationship between advanced paternal age and increased ASD risk has been established in different studies [67], and de novo SNVs were indicated as potential risk factors [68]. Notably, a sibling of the A36V carrier identified in the present study also was diagnosed with SCZ; therefore, one or more additional factors (genetic or environmental) may contribute to increased susceptibility to SCZ in this particular family, a conjecture that may be explained, in part, by a two-hit model [69].

Given the potentially important role of de novo SNVs in SCZ/ASD susceptibility, we tested for the presence of candidate SNVs in the parents of the carrier patients, if parental DNA samples were available. We discovered one de novo missense SNV (p.A36V in CACNA1C) in a patient with SCZ, and showed (by electrophysiological recording) that cells expressing the $\mathrm{A} 36 \mathrm{~V}$ neuronal $\mathrm{Ca}_{\mathrm{v}} 1.2$ channel exhibit slower decreases in $\mathrm{Ca}^{2+}$ current than do cells expressing the WT channel. This finding supports the hypothesis that this de novo missense SNV is an ultra-rare disruptive SNV affecting CDI, possibly contributing to susceptibility to psychiatric disorders. The $\mathrm{Ca}^{2+}$ dependent signaling pathway is a key component in neural circuit formation, gene expression, and neuronal plasticity [70, 71]. In addition to the excessive $\mathrm{Ca}^{2+}$ entry per se, disturbance of intracellular $\mathrm{Ca}^{2+}$ homeostasis dynamics may lead to neural dysfunction by the dis-regulation of downstream signaling pathways that also are associated with SCZ/ASD [72].

Several limitations should be considered when interpreting the results of our study. First, the lack of statistical significance in our association study may reflect the small sample size; a new study in a larger cohort is needed. Nonetheless, our data revealed that these SNVs are ultra-rare in the Japanese population. These genetic data may be incorporated into future large-scale WESs, potentially supporting the hypothesis that an increased burden of ultra-rare deleterious mutations is observed in patients with SCZ/ASD. Second, due to the lack of enough parental DNA samples, we were able to perform segregation analysis for only a few of the prioritized SNVs. To identify more de novo mutations associated with susceptivity to SCZ/ ASD, we will need to collect more DNA samples of family members and perform trios-based exome-sequencing studies. Finally, our sequence analysis did not cover the promoters, untranslated regions, or intronic regions of the target genes, which may contain important mutations at regulatory sites.

In conclusion, we identified 1 nonsense SNV (p.C1451* in CACNA1D), 1 de novo SNV (p.A36V in CACNA1C), 1 rare short deletion (p.E1675del in CACNA1D), and 14 NSstrict SNVs by genetic analysis of the CANCA1C, CACNA1D, CACNA11, and CACNA1S genes in humans. Our genetic and biological data imply that the de novo SNV (p.A36V in CACNA1C) may increase susceptibility to $\mathrm{SCZ}$ pathogenesis by perturbing $\mathrm{CDI}$. Furthermore, our data provide evidence in support of the potential role of VGCC-encoding genes in psychiatric disorders.

\section{REFERENCES}

1. Saha S, Chant D, McGrath J. A systematic review of mortality in schizophrenia. Arch Gen Psychiatry. 2007;64:1123.

2. Lai M-C, Lombardo MV, Baron-Cohen S. Autism. Lancet (Lond, Engl). 2014;383:896-910.

3. Lichtenstein P, Yip BH, Björk C, Pawitan Y, Cannon TD, Sullivan PF, et al. Common genetic determinants of schizophrenia and bipolar disorder in Swedish families: a population-based study. Lancet (Lond, Engl). 2009;373:234-9.

4. Colvert E, Tick B, McEwen F, Stewart C, Curran SR, Woodhouse E, et al. Heritability of autism spectrum disorder in a UK population-based twin sample. JAMA Psychiatry. 2015;72:415-23.

5. Purcell SM, Moran JL, Fromer M, Ruderfer $D$, Solovieff $N$, Roussos $P$, et al. A polygenic burden of rare disruptive mutations in schizophrenia. Nature 2014;506:185-90.

6. Ripke S, Neale BM, Corvin A, Walters JTR, Farh KH, Holmans PA, et al. Biological insights from 108 schizophrenia-associated genetic loci. Nature 2014;511:421-7.

7. Gaugler T, Klei L, Sanders SJ, Bodea CA, Goldberg AP, Lee AB, et al. Most genetic risk for autism resides with common variation. Nat Genet. 2014;46:881-5.

8. Geschwind DH, Flint J. Genetics and genomics of psychiatric disease. Science 2015;349:1489-94.

9. Simms BA, Zamponi GW. Neuronal voltage-gated calcium channels: structure, function, and dysfunction. Neuron 2014;82:24-45.

10. Hall J, Trent S, Thomas KL, O'Donovan MC, Owen MJ. Genetic risk for schizophrenia: convergence on synaptic pathways involved in plasticity. Biol Psychiatry. 2015;77:52-58.

11. Moon AL, Haan N, Wilkinson LS, Thomas KL, Hall J. CACNA1C: association with psychiatric disorders, behavior, and neurogenesis. Schizophr Bull. 2018;44:958-65.

12. Bhat S, Dao DT, Terrillion CE, Arad M, Smith RJ, Soldatov NM, et al. CACNA1C (Cav1.2) in the pathophysiology of psychiatric disease. Prog Neurobiol. 2012;99:1-14.

13. Striessnig J, Pinggera A, Kaur G, Bock G, Tuluc P. L-type $\mathrm{Ca}(2+)$ channels in heart and brain. Wiley Interdiscip Rev Membr Transp Signal. 2014;3:15-38.

14. Splawski I, Timothy KW, Sharpe LM, Decher N, Kumar P, Bloise R, et al. CaV1.2 calcium channel dysfunction causes a multisystem disorder including arrhythmia and autism. Cell 2004;119:19-31.

15. Splawski I, Timothy KW, Decher N, Kumar P, Sachse FB, Beggs AH, et al. Severe arrhythmia disorder caused by cardiac L-type calcium channel mutations. Proc Natl Acad Sci USA. 2005;7:8089-96.

16. Bader PL, Faizi M, Kim LH, Owen SF, Tadross MR, Alfa RW, et al. Mouse model of Timothy syndrome recapitulates triad of autistic traits. Proc Natl Acad Sci USA. 2011;108:15432. 
17. Rendall AR, Ford AL, Perrino PA, Holly, Fitch R. Auditory processing enhancements in the TS2-neo mouse model of timothy syndrome, a rare genetic disorder associated with autism spectrum disorders. Adv Neurodev Disord. 2017;1:176-89.

18. Horigane S, Ozawa Y, Zhang J, Todoroki H, Miao P, Haijima A, et al. A mouse model of Timothy syndrome exhibits altered social competitive dominance and inhibitory neuron development. FEBS Open Bio. 2020;10:1436-46.

19. Li J, Zhao L, You Y, Lu T, Jia M, Yu H, et al. Schizophrenia related variants in CACNA1C also confer risk of autism. PLoS ONE. 2015;10:e0133247.

20. Roussos P, Mitchell AC, Voloudakis G, Fullard JF, Pothula VM, Tsang J, et al. A role for noncoding variation in schizophrenia. Cell Rep. 2014;9:1417-29.

21. Splawski I, Yoo DS, Stotz SC, Cherry A, Clapham DE, Keating MT. CACNA1H mutations in autism spectrum disorders. J Biol Chem. 2006;281:22085-91.

22. Cross-Disorder Group of the Psychiatric Genomics. Identification of risk loci with shared effects on five major psychiatric disorders: a genome-wide analysis. Lancet. 2013;381:1371-9.

23. Pinggera A, Lieb A, Benedetti B, Lampert M, Monteleone S, Liedl KR, et al. CACNA1D de novo mutations in autism spectrum disorders activate cav1.3 I-type calcium channels. Biol Psychiatry. 2015;77:816-22.

24. Ripke S, Sanders AR, Kendler KS, Levinson DF, Sklar P, Consortium TSPG-WAS (GWAS). et al. Genome-wide association study identifies five new schizophrenia loci. Nat Genet. 2011;43:969-76.

25. Sklar P, Ripke S, Scott LJ, Andreassen OA, Cichon S, Group PGCBDW. et al. Largescale genome-wide association analysis of bipolar disorder identifies a new susceptibility locus near ODZ4. Nat Genet. 2011;43:977-83.

26. De Rubeis S, He X, Goldberg AP, Poultney CS, Samocha K, Cicek AE, et al. Synaptic, transcriptional and chromatin genes disrupted in autism. Nature 2014;515:209-15.

27. Xu W, Liu Y, Chen J, Guo Q, Liu K, Wen Z, et al. Genetic risk between the CACNA1I gene and schizophrenia in Chinese Uygur population. Hereditas 2018;155:5.

28. Lu AT-H, Dai X, Martinez-Agosto JA, Cantor RM. Support for calcium channel gene defects in autism spectrum disorders. Mol Autism. 2012;3:18.

29. Andrade A, Hope J, Allen A, Yorgan V, Lipscombe D, Pan JQ. A rare schizophrenia risk variant of CACNA1I disrupts CaV3.3 channel activity. Sci Rep. 2016;6:34233.

30. Adzhubei IA, Schmidt S, Peshkin L, Ramensky VE, Gerasimova A, Bork $P$, et al. A method and server for predicting damaging missense mutations. Nat Methods. 2010;7:248-9.

31. Chun S, Fay JC. Identification of deleterious mutations within three human genomes. Genome Res. 2009;19:1553-61.

32. Schwarz JM, Rödelsperger C, Schuelke M, Seelow D. MutationTaster evaluates disease-causing potential of sequence alterations. Nat Methods. 2010;7:575-6.

33. Kumar P, Henikoff $S, \mathrm{Ng} P C$. Predicting the effects of coding non-synonymous variants on protein function using the SIFT algorithm. Nat Protoc. 2009;4:1073-81.

34. Kamijo S, Ishii Y, Horigane SI, Suzuki K, Ohkura M, Nakai J, et al. A critical neurodevelopmental role for I-type voltage-gated calcium channels in neurite extension and radial migration. J Neurosci. 2018;38:5551-66.

35. Tyagi R, Lai R, Duggleby RG. A new approach to 'megaprimer' polymerase chain reaction mutagenesis without an intermediate gel purification step. BMC Biotechnol. 2004;4:2

36. Altschul SF, Madden TL, Schäffer AA, Zhang J, Zhang Z, Miller W, et al. Gapped BLAST and PSI-BLAST: a new generation of protein database search programs. Nucleic Acids Res. 1997;25:3389-402.

37. Kawabata T. HOMCOS: an updated server to search and model complex 3D structures. J Struct Funct Genomics. 2016;17:83-99.

38. Wu J, Yan Z, Li Z, Qian X, Lu S, Dong M. et al. Structure of the voltage-gated calcium channel Cav1.1 at $3.6 \AA$ A resolution. Nature. 2016;537:191-6.

39. Shen $\mathrm{H}$, Li Z, Jiang Y, Pan X, Wu J, Cristofori-Armstrong B, et al. Structural basis for the modulation of voltage-gated sodium channels by animal toxins. Science. 2018;362.

40. Liu Z, Vogel HJ. Structural basis for the regulation of L-type voltage-gated calcium channels: interactions between the $\mathrm{N}$-terminal cytoplasmic domain and $\mathrm{Ca} 2$ +-calmodulin. Front Mol Neurosci. 2012;5:1-46.

41. Jones DT, Cozzetto D. DISOPRED3: precise disordered region predictions with annotated protein-binding activity. Bioinformatics 2015;31:857-63.

42. Kawabata T, Nishikawa K. Protein structure comparison using the Markov transition model of evolution. Proteins 2000;41:108-22.

43. Šali A, Blundell TL. Comparative protein modelling by satisfaction of spatial restraints. J Mol Biol. 1993;234:779-815.

44. Case DA, Aktulga HM, Belfon K, Ben-Shalom IY, Brozell SR, Cerutti DS, et al. Amber 2021. Univ California, San Fr. 2021;1-195.

45. Kiyonaka S, Wakamori M, Miki T, Uriu Y, Nonaka M, Bito $H$, et al. RIM1 confers sustained activity and neurotransmitter vesicle anchoring to presynaptic $\mathrm{Ca} 2+$ channels. Nat Neurosci. 2007;10:691-701.

46. Hamill OP, Marty A, Neher E, Sakmann B, Sigworth FJ. Improved patch-clamp techniques for high-resolution current recording from cells and cell-free membrane patches. Pflug Arch. 1981;391:85-100.
47. Wakamori M, Yamazaki K, Matsunodaira H, Teramoto T, Tanaka I, Niidome T, et al Single tottering mutations responsible for the neuropathic phenotype of the P-type calcium channel. J Biol Chem. 1998;273:34857-67.

48. Antzelevitch C, Pollevick GD, Cordeiro JM, Casis O, Sanguinetti MC, Aizawa Y, et al. Loss-of-function mutations in the cardiac calcium channel underlie a new clinical entity characterized by ST-segment elevation, short QT intervals, and sudden cardiac death. Circulation 2007;115:442-9.

49. Simms BA, Zamponi GW. The Brugada syndrome mutation A39V does not affect surface expression of neuronal rat Cav1.2 channels. Mol Brain. 2012;5:9.

50. Ben-Johny M, Yue DT. Calmodulin regulation (calmodulation) of voltage-gated calcium channels. J Gen Physiol. 2014;143:679-92.

51. Ben-Johny M, E. Dick I, Sang L, B. Limpitikul W, Wei Kang P, Niu J, et al. Towards a unified theory of calmodulin regulation (calmodulation) of voltage-gated calcium and sodium channels. Curr Mol Pharm. 2015;8:188.

52. Simms BA, Souza IA, Zamponi GW. A novel calmodulin site in the Cav1.2 $\mathrm{N}$-terminus regulates calcium-dependent inactivation. Pflug Arch Eur J Physiol. 2014;466:1793-803.

53. Dick IE, Tadross MR, Liang H, Tay LH, Yang W, Yue DT. A modular switch for spatial $\mathrm{Ca} 2+$ selectivity in the calmodulin regulation of $\mathrm{CaV}$ channels. Nature 2008;451:830-4.

54. Simms BA, Souza I, Zamponi GW. Effect of the Brugada syndrome mutation A39V on calmodulin regulation of Cav1.2 channels. Mol Brain. 2014;7:34.

55. Casamassima F, Hay AC, Benedetti A, Lattanzi L, Cassano GB, Perlis RH. L-type calcium channels and psychiatric disorders: a brief review. Am J Med Genet Part B Neuropsychiatr Genet. 2010;153:1373-90.

56. Dirksen RT, Nakai J, Gonzalez A, Imoto K, Beam KG. The S5-S6 linker of repeat I is a critical determinant of L-type $\mathrm{Ca} 2+$ channel conductance. Biophys J. 1997;73:1402-9.

57. Findeisen F, Minor DL. Progress in the structural understanding of voltage-gated calcium channel (CaV) function and modulation. Channels (Austin). 2010;4:459.

58. Yoshimizu T, Pan JQ, Mungenast AE, Madison JM, Su S, Ketterman J, et al Functional implications of a psychiatric risk variant within CACNA1C in induced human neurons. Mol Psychiatry. 2015;20:162-9.

59. Bigos KL, Mattay VS, Callicott JH, Straub RE, Vakkalanka R, Kolachana B, et al. Genetic variation in CACNA1C affects brain circuitries related to mental illness. Arch Gen Psychiatry. 2010;67:939-45.

60. Bartels $P, Y u$ D, Huang $H, H u Z$, Herzig $S$, Soong TW. Alternative splicing at $N$ terminus and domain I modulates CaV1.2 inactivation and surface expression. Biophys J. 2018;114:2095-106.

61. Liao P, Tan FY, Mui CL, Yue DT, Tuck WS. Splicing for alternative structures of Cav1.2 Ca2+ channels in cardiac and smooth muscles. Cardiovasc Res. 2005;68:197-203.

62. Arikkath J, Campbell KP. Auxiliary subunits: essential components of the voltagegated calcium channel complex. Curr Opin Neurobiol. 2003;13:298-307.

63. Wang W, Corominas R, Lin GN. De novo mutations from whole exome sequencing in neurodevelopmental and psychiatric disorders: from discovery to application. Front Genet. 2019;10:258.

64. Howrigan DP, Rose SA, Samocha KE, Fromer M, Cerrato F, Chen WJ, et al. Exome sequencing in schizophrenia-affected parent-offspring trios reveals risk conferred by protein-coding de novo mutations. Nat Neurosci.2020;23:185-9.

65. Ambalavanan A, Girard SL, Ahn K, Zhou S, Dionne-Laporte A, Spiegelman D, et al. De novo variants in sporadic cases of childhood onset schizophrenia. Eur J Hum Genet. 2016;24:944-8.

66. Fromer M, Pocklington AJ, Kavanagh DH, Williams HJ, Dwyer S, Gormley $\mathrm{P}$, et al. De novo mutations in schizophrenia implicate synaptic networks. Nature 2014;506:179-84

67. de Kluiver H, Buizer-Voskamp JE, Dolan CV, Boomsma DI. Paternal age and psychiatric disorders: a review. Am J Med Genet B Neuropsychiatr Genet. 2017;174:202-13.

68. Janecka M, Mill J, Basson MA, Goriely A, Spiers H, Reichenberg A, et al. Advanced paternal age effects in neurodevelopmental disorders-review of potential underlying mechanisms. Transl Psychiatry. 2017;7:e1019.

69. Maynard TM, Sikich L, Lieberman JA, LaMantia AS. Neural development, cell-cell signaling, and the "Two-Hit" hypothesis of schizophrenia. Schizophr Bull. 2001;27:457-76

70. Flavell SW, Greenberg ME. Signaling mechanisms linking neuronal activity to gene expression and plasticity of the nervous system. Annu Rev Neurosci. 2008;31:563-90

71. Spitzer NC. Electrical activity in early neuronal development. Nature 2006;444:707-12.

72. Ebert DH, Greenberg ME. Activity-dependent neuronal signalling and autism spectrum disorder. Nat 2013 4937432. 2013;493:327-37.

73. Bekker GJ, Kawabata T, Kurisu G. The Biological Structure Model Archive (BSMArc): an archive for in silico models and simulations. Biophys Rev. 2020;12:371-5. 
74. Pettersen EF, Goddard TD, Huang CC, Couch GS, Greenblatt DM, Meng EC, et al. UCSF Chimera-a visualization system for exploratory research and analysis. J Comput Chem. 2004;25:1605-12.

\section{ACKNOWLEDGEMENTS}

We thank Dr. Yasuo Mori for providing the BHK- $a_{2} \delta+\beta_{2}$ cells. This work was supported by JSPS KAKENHI Grant Numbers JP2OK16490 (S.H.), JP2OH03339, JP21H05091 (S.T.-K.), JP19H01007 (H.B.), and JP16H06276 (AdAMS to H.B. and S.T.K.); the Ministry of Education, Culture, Sports, Science and Technology of Japan; the Ministry of Health, Labour and Welfare of Japan; the Strategic Research Program for Brain Sciences from the Japan Agency for Medical Research and Development (AMED) under Grant Numbers JP20dm0107131 (M.W.), JP20dm0107132 (H.F.), JP20dm0107130 (B.A. and S.T.-K.), JP20dm0107087, JP20dm0207075, and JP20ak0101113 (N.O.); the Brain Mapping by Integrated Neurotechnologies for Disease Studies (Brain/MINDS) from AMED; the Innovative Areas "Glial assembly: a new regulatory machinery of brain function and disorders" program; the Innovative Areas "Comprehensive Brain Science Network" program; the Platform Project for Supporting Drug Discovery and Life Science Research (Basis for Supporting Innovative Drug Discovery and Life Science Research (BINDS)) from AMED under Grant Number JP21am0101066 (Support Number 0305) (T.K.); the Takeda Science Foundation (S.H.); the Toray Science Foundation (S.T.-K.); JST-Mirai Program Grant Number JPMJMI21G6 (S.T.-K.); and the Toyoaki Scholarship Foundation (S.H., S.T.-K.). We also thank the patients and their families for participating in this study. The model structures have been submitted to the Biological Structure Model Archive (BSM-Arc) under BSM-ID BSM00025 (https://bsma.pdbj.org/entry/25) [73].

\section{AUTHOR CONTRIBUTIONS}

$\mathrm{CW}, \mathrm{SIH}, \mathrm{MW}, \mathrm{SU}, \mathrm{TK}, \mathrm{HF}, \mathrm{BA}, \mathrm{HB}$ and STK contributed to conception and design. CW, $\mathrm{SIH}, \mathrm{MW}, \mathrm{SU}, \mathrm{TK}, \mathrm{HF}, \mathrm{IK}, \mathrm{HK}, \mathrm{KI}, \mathrm{YN}, \mathrm{YI}, \mathrm{MI}, \mathrm{NI}, \mathrm{TO}, \mathrm{BA}, \mathrm{DM}, \mathrm{TYd}, \mathrm{HB}, \mathrm{TYK}, \mathrm{STK}$ and NO contributed to data acquisition and pre-processing. CW, SIH, MW, SU, TK, HF and BA analyzed the data and drafted the manuscript. All authors participated in revising it critically for important intellectual content.

\section{COMPETING INTERESTS}

The authors declare no competing interests.

\section{ADDITIONAL INFORMATION}

Supplementary information The online version contains supplementary material available at https://doi.org/10.1038/s41398-022-01851-y.

Correspondence and requests for materials should be addressed to Branko Aleksic or Sayaka Takemoto-Kimura.

Reprints and permission information is available at http://www.nature.com/ reprints

Publisher's note Springer Nature remains neutral with regard to jurisdictional claims in published maps and institutional affiliations.

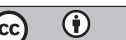

Open Access This article is licensed under a Creative Commons Attribution 4.0 International License, which permits use, sharing, adaptation, distribution and reproduction in any medium or format, as long as you give appropriate credit to the original author(s) and the source, provide a link to the Creative Commons license, and indicate if changes were made. The images or other third party material in this article are included in the article's Creative Commons license, unless indicated otherwise in a credit line to the material. If material is not included in the article's Creative Commons license and your intended use is not permitted by statutory regulation or exceeds the permitted use, you will need to obtain permission directly from the copyright holder. To view a copy of this license, visit http://creativecommons. org/licenses/by/4.0/.

(c) The Author(s) 2022 